Review

\title{
Relativistic effects in spectroscopy and photophysics of heavy-metal complexes illustrated by spin-orbit calculations of $\left[\operatorname{Re}(\text { imidazole })(\mathrm{CO})_{3}(\text { phen })\right]^{+}$
}

\author{
Radka Bakováa,b, Majed Cherguic, Chantal Danield ${ }^{\mathrm{d}}$, Antonín Vlček Jr. ${ }^{\mathrm{a}, \mathrm{e}, *}$, Stanislav Záliša, ${ }^{a * *}$ \\ a J. Heyrovský Institute of Physical Chemistry, Academy of Sciences of the Czech Republic, Dolejškova 3, CZ-182 23 Prague 8, Czech Republic \\ ${ }^{b}$ Department of Physical Chemistry and Macromolecular Chemistry, Faculty of Science, Charles University, Albertov 6, 12843 Prague 2, Czech Republic \\ ' Laboratoire de Spectroscopie Ultrarapide, ISIC, FSB-BSP, Ecole Polytechnique Fédérale de Lausanne, CH-1015 Lausanne-Dorigny, Switzerland \\ d Laboratoire de Chimie Quantique, Institut de Chimie de Strasbourg, UMR7177, CNRS-Université de Strasbourg, 4 Rue Blaise Pascal, CS 90032, F-67081 Strasbourg-Cedex, France \\ e Queen Mary University of London, School of Biological and Chemical Sciences, Mile End Road, London E1 4NS, United Kingdom
}

\section{Contents}

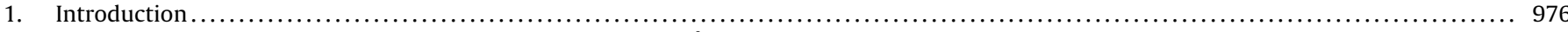

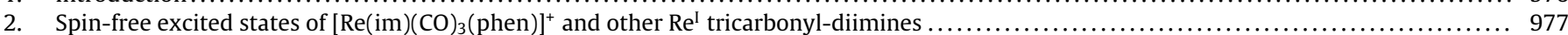

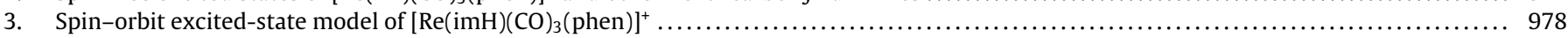

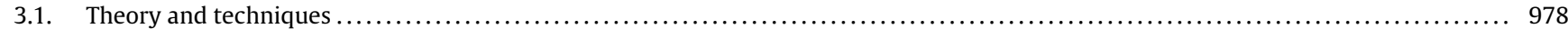

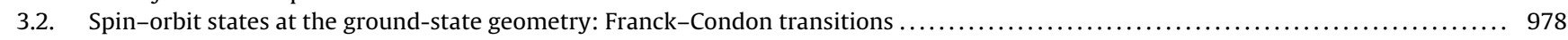

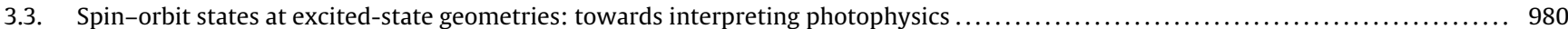

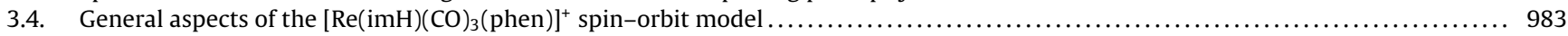

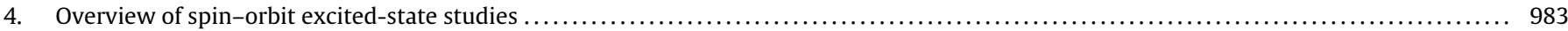

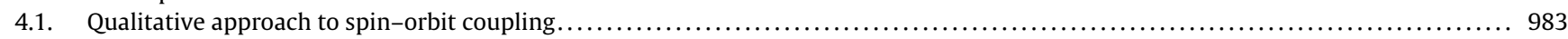

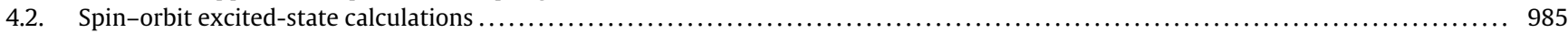

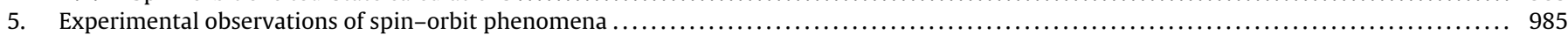

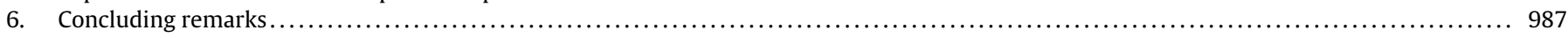

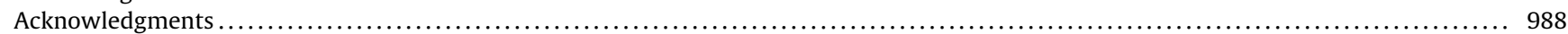

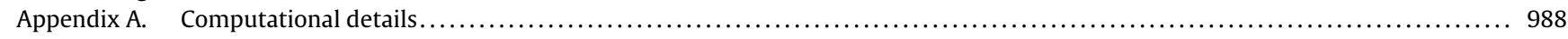

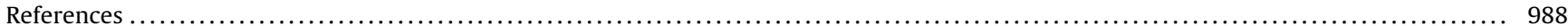

\section{A R T I C L E I N F O}

\section{Article history:}

Received 19 November 2010

Accepted 28 December 2010

Available online 11 January 2011

\begin{abstract}
A B S T R A C T
Spin-orbit coupling (SOC) is an essential factor in photophysics of heavy transition metal complexes. By enabling efficient population of the lowest triplet state and its strong emission, it gives rise to a very interesting photophysical behavior and underlies photonic applications such as organic light emitting diodes (OLED) or luminescent imaging agents. SOC affects excitedstate characters, relaxation dynamics, radiative and nonradiative decay pathways, as well as
\end{abstract}

\footnotetext{
Abbreviations: ADF, Amsterdam Density Functional (quantum chemistry software package); bpy, 2,2'-bipyridine; $\mathrm{C}^{\wedge} \mathrm{N}$, cyclometallated ligands coordinated by an

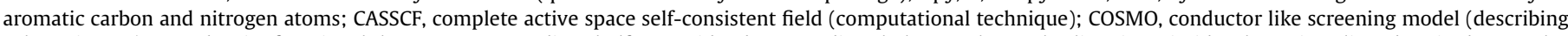

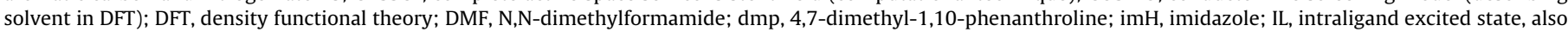

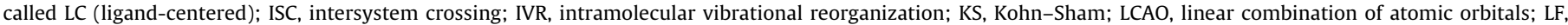

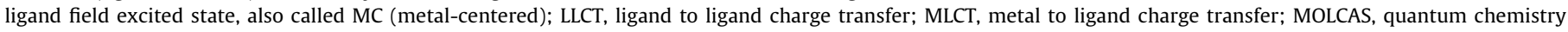

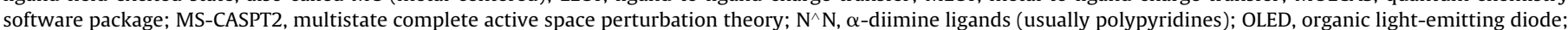

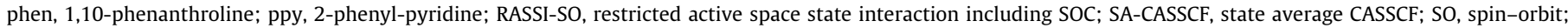

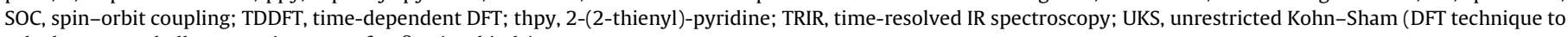
calculate open-shell systems in terms of $\alpha, \beta$ spinorbitals).

* Corresponding author at: Queen Mary University of London, School of Biological and Chemical Sciences, J. Priestley Bldg., Mile End Road, London E1 4NS, United Kingdom. Tel.: +44 (0) 207882 3260; fax: +44 (0) 2078827427.

** Corresponding author. Tel: +420 26605 3268; fax: +420 286582307.

E-mail addresses: a.vlcek@qmul.ac.uk (A. Vlček Jr.), zalis@jh-inst.cas.cz (S. Záliš). 


\section{Keywords:}

Rhenium

Carbonyl

Diimine

Spin-orbit coupling

TDDFT

Photophysics

Spectroscopy

Excited states

Ultrafast dynamics

Density Functional Theory

CASSCF

MS-CASPT2 lifetimes and reactivity. We present a new photophysical model based on mixed-spin states, illustrated by relativistic spin-orbit TDDFT and MS-CASPT2 calculations of $\left[\operatorname{Re}(\right.$ imidazole $)(C O)_{3}(1,10-$ phenanthroline) $]^{+}$. An excited-state scheme is constructed from spin-orbit (SO) states characterized by their energies, double-group symmetries, parentages in terms of contributing spin-free singlets and triplets, and oscillator strengths of corresponding transitions from the ground state. Some of the predictions of the relativistic SO model on the number and nature of the optically populated and intermediate excited states are qualitatively different from the spin-free model. The relativistic excited-state mode accounts well for electronic absorption and emission spectra of $\operatorname{Re}^{\mathrm{I}}$ carbonyl diimines, as well as their complex photophysical behavior. Then, we discuss the SO aspects of photophysics of heavy metal complexes from a broader perspective. Qualitative SO models as well as previous relativistic excited-state calculations are briefly reviewed together with experimental manifestations of SOC in polypyridine and cyclometallated complexes of second- and third row $\mathrm{d}^{6}$ metals. It is shown that the relativistic SO model can provide a comprehensive and unifying photophysical picture.

(C) 2011 Elsevier B.V. All rights reserved.

\section{Introduction}

Efficient population of low-lying triplet state(s) and intense phosphorescence are the two phenomena that distinguish photophysics of heavy transition metal complexes from those of organic compounds. They are at heart of important photonic applications of transition metal complexes as luminophores in "organic light-emitting diodes" (OLED), sensors, probes, imaging agents, and photosensitizers of electron or energy transfer. Both these effects are consequences of spin-orbit coupling (SOC) introduced by the metal atom, whose electrons are involved in relevant excitations [1-3]. SOC accelerates intersystem crossing (ISC) from optically populated "singlet" excited states into "triplet" states, and makes the "triplet" radiative decay allowed and relatively fast. However, the previous statement is somewhat self-contradictory since assigning spin labels to electronic states is not appropriate in the presence of strong SOC that, to a large extent, smears off the distinctions between fluorescence and phosphorescence as well as between intersystem crossing and internal conversion. The "appropriateness of assigning spin labels to excited states of inorganic complexes" was questioned [4] already in 1974 , shortly after the discovery of $\left[\mathrm{Ru}(\mathrm{bpy})_{3}\right]^{2+}$ emission and excited-state electron-transfer reactivity. Distinct "triplet-state" photophysics and photochemistry are especially eminent in the case of closed-shell pseudo-octahedral $\mathrm{d}^{6}$, square-planar $\mathrm{d}^{8}$ and flattened-tetrahedral $\mathrm{d}^{10}$ metal complexes with electron-accepting $\alpha$-diimine $\left(\mathrm{N}^{\wedge} \mathrm{N}\right)$ or cyclometallated $\left(\mathrm{C}^{\wedge} \mathrm{N}\right)$ ligands that introduce low-lying metal to ligand charge transfer (MLCT, $\mathrm{d} \pi^{*}$ ) and intraligand (IL, $\pi \pi^{*}$ ) excited states, while the metal atom (usually $\mathrm{Re}^{\mathrm{I}}$, $\mathrm{Os}^{\mathrm{II}}, \mathrm{Ir}^{\mathrm{III}}, \mathrm{Pt}^{\mathrm{II}}$ or $\left.\mathrm{Au}^{\mathrm{I}}\right)$ is responsible for the relativistic SO effects.

Despite the essential role of SOC in spectroscopy and photophysics of heavy metal complexes, experimental observations on excited-state properties and dynamics are usually interpreted at the level of spin-free singlet and triplet states, arguing that the large difference in their lifetimes and small magnitudes of SOCinduced triplet splitting (i.e. zero-field splitting, ZFS) warrant the neglect of SOC effects, at least at ambient temperatures. Quantum chemical calculations of excited states also often neglect SOC. Nevertheless, spin-free CASSCF/CASPT2-type [5-8] and (TD)DFT [8-10] techniques are still instrumental in interpreting photophysical behavior and spectra. DFT techniques with hybrid functionals are successfully used to visualize singlet and triplet CT states and reveal their characters by displaying differences of electron density distribution upon excitation and/or excited-state spin-density distributions [9-13]. In such studies, the UKS technique is employed to optimize the lowest triplet-state structures, which are then used to calculate excited-state IR and UV-vis absorption spectra $[9,11,14,15]$. Spin-free excited-state structures can also be optimized using TDDFT. Unlike UKS, TDDFT is not limited to the lowest state of particular spin and symmetry [9].

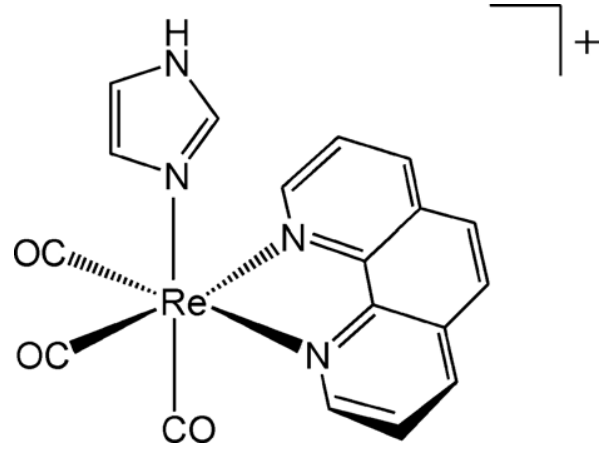

Fig. 1. Schematic structure of $\left[\operatorname{Re}(\mathrm{imH})(\mathrm{CO})_{3}(\text { phen })\right]^{+}$.

Neglecting SOC is, however, a severe approximation that could miss important effects and lead to incomplete, if not incorrect, interpretations of spectroscopic, photophysical and photochemical observations. SO effects can be introduced at various conceptual and computational levels. To account for the strong phosphorescence, it is sufficient to consider SO as a weak perturbation that splits the lowest triplet state into three spin components and makes some of them emissive by mixing with allowed singlets [1-3]. This approach aids developing OLED luminophores and has been used to formulate useful design rules $[1-3,16,17]$. On the other hand, assigning UV-vis absorption spectra and understanding photophysical behavior requires us to go one step further and think in terms of spin-orbit states characterized by their double-group symmetry but no spin labels [18-20].

In this paper, we develop and explain an SO excited-state model of $\left[\operatorname{Re}(\mathrm{imH})(\mathrm{CO})_{3}(\text { phen })\right]^{+}$(Fig. 1) using SO TDDFT and CASSCF/CASPT2-type calculations, and examine how this new approach can interpret available photophysical data. It will be demonstrated that the SO model accounts very well for absorption spectra, emission properties, and excited-state dynamics, some of its conclusions being qualitatively different from the spinfree approach. $\left[\operatorname{Re}(\mathrm{imH})(\mathrm{CO})_{3} \text { (phen) }\right]^{+}$represents a broad class of $\operatorname{Re}^{\mathrm{I}}$ tricarbonyl-diimine complexes [21-23], which show very rich photophysics and photochemistry $[10,14,15,24]$ and engage in a range of photonic applications such as photosensitizers and phototriggers of electron-transfer reactions [11,25-29], photocatalysts of $\mathrm{CO}_{2}$ reduction [30-32], phosphorescent labels and probes of biomolecules [33-36], sensors [37,38], molecular switches [39-42] and OLED emitters [43], or probes of ps-ns dynamics of solvents, proteins or supramolecular hosts $[11,24,50,51,63-65]$. The chosen example $\left[\operatorname{Re}(\mathrm{imH})(\mathrm{CO})_{3}(\text { phen })\right]^{+}[44]$ not only epitomizes the salient features of $\mathrm{Re}^{\mathrm{I}}$ carbonyl-diimine photophysics but also has a prominent position amongst Re-based photosensitizers because of its ability to trigger photoinduced electron transfer and relaxation 


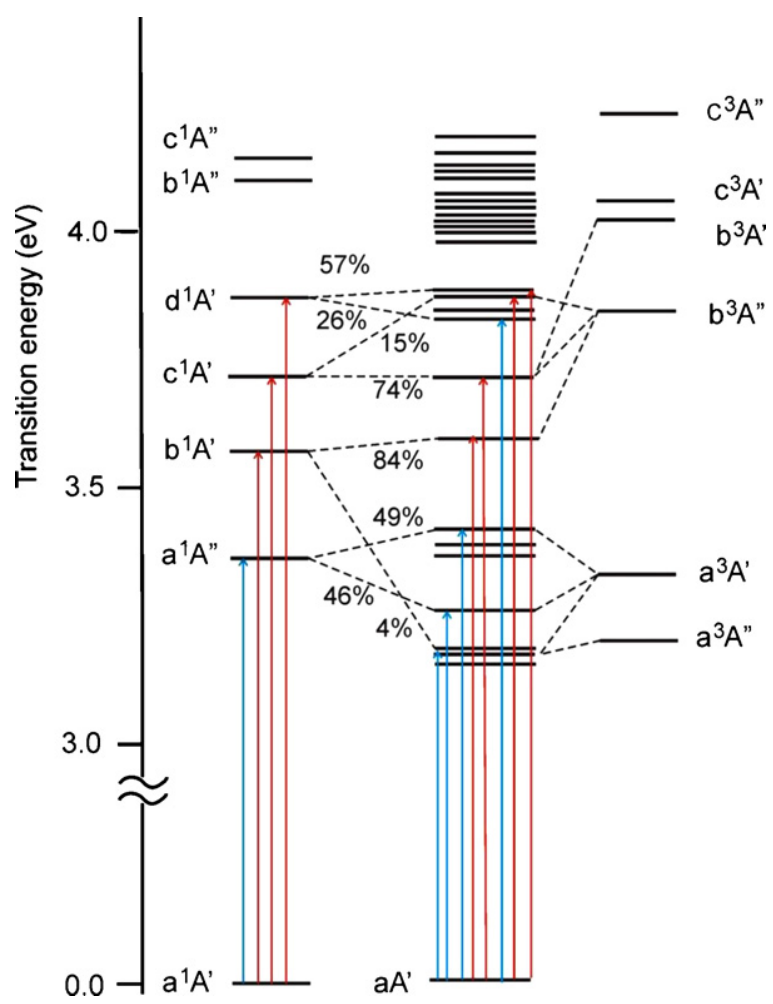

Fig. 2. Correlation of MS-CASPT2 calculated singlet (left) and triplet (right) spinfree states with SO states (middle) of $\left[\operatorname{Re}(\mathrm{imH})(\mathrm{CO})_{3}(\text { phen })\right]^{+}$in vacuo. Red, blue, and black arrows indicate transitions with oscillator strengths larger than 0.01 , 0.001-0.01, and 0.0005-0.001, respectively. RASSI-SO MS-CASPT2 calculation on CASSCF wavefunctions.

dynamics in Re-labeled proteins [11,25,44-54]. The proposed relativistic SO model accounts well for the excited-state behavior of $\operatorname{Re}^{\mathrm{I}}$ carbonyl diimines and explains in a unified way various aspects of spectroscopy, photophysics and photochemistry of heavy metal complexes at large.

\section{Spin-free excited states of $\left[\operatorname{Re}(\mathrm{im})(\mathrm{CO})_{3}(\text { phen })\right]^{+}$and other $\operatorname{Re}^{\mathrm{I}}$ tricarbonyl-diimines}

In the spin-free model, electronic states are classified as spin singlets $\left(S=0, M_{S}=1\right)$ and triplets $\left(S=1, M_{S}=3\right)$, which are shown in the left and right columns, respectively, of Figs. 2 and 3. Regardless the spin, the spectroscopically and photophysically relevant low-lying excited states of $\operatorname{Re}^{\mathrm{I}}$ tricarbonyl diimines $\left[\operatorname{Re}(\mathrm{L})(\mathrm{CO})_{3}\left(\mathrm{~N}^{\wedge} \mathrm{N}\right)\right]^{\mathrm{n}}$ result from mixing of MLCT, LLCT, and IL characters [21,22], whose extent determines the excited-state properties. Two types of mixing have to be considered:

(i) MLCT-LLCT mixing. The lowest states are predominantly MLCT but contain contributions from ligand-to-ligand charge transfer (LLCT) due to a d-orbital mixing with $\pi^{*}(\mathrm{CO})$ and occupied $\pi(\mathrm{L})$ orbitals. Thus, MLCT states always involve depopulation of carbonyl $\pi^{*}$ orbitals and should be viewed as $\operatorname{Re}^{\mathrm{I}}(\mathrm{CO})_{3} \rightarrow$ bpy CT. The $\pi^{*}(\mathrm{CO})$ electronic depopulation upon excitation is clearly manifested by shifts of $v(\mathrm{CO})$ vibrations to higher energies [9,12-14,22,24,55-59]. Mixing of MLCT and $L \rightarrow \mathrm{N}^{\wedge} \mathrm{N}$ LLCT characters is especially strong in complexes containing $\pi$ donating ligand L (halides, $\mathrm{NCS}^{-}$, amides, phosphides, alkoxides, thiolates, imidazole, etc.) $[9-13,21,22,60,61]$. For example, the low-lying excited-states of $\left[\operatorname{Re}(\mathrm{I})(\mathrm{CO})_{3}\right.$ (bpy)] contain about $50 \%$ of $\mathrm{I} \rightarrow$ bpy LLCT and $50 \% \operatorname{Re}(\mathrm{CO})_{3} \rightarrow$ bpy MLCT characters [10] and are best viewed as delocalized $\operatorname{Re}(\mathrm{I})(\mathrm{CO})_{3} \rightarrow$ bpy CT. Mixing

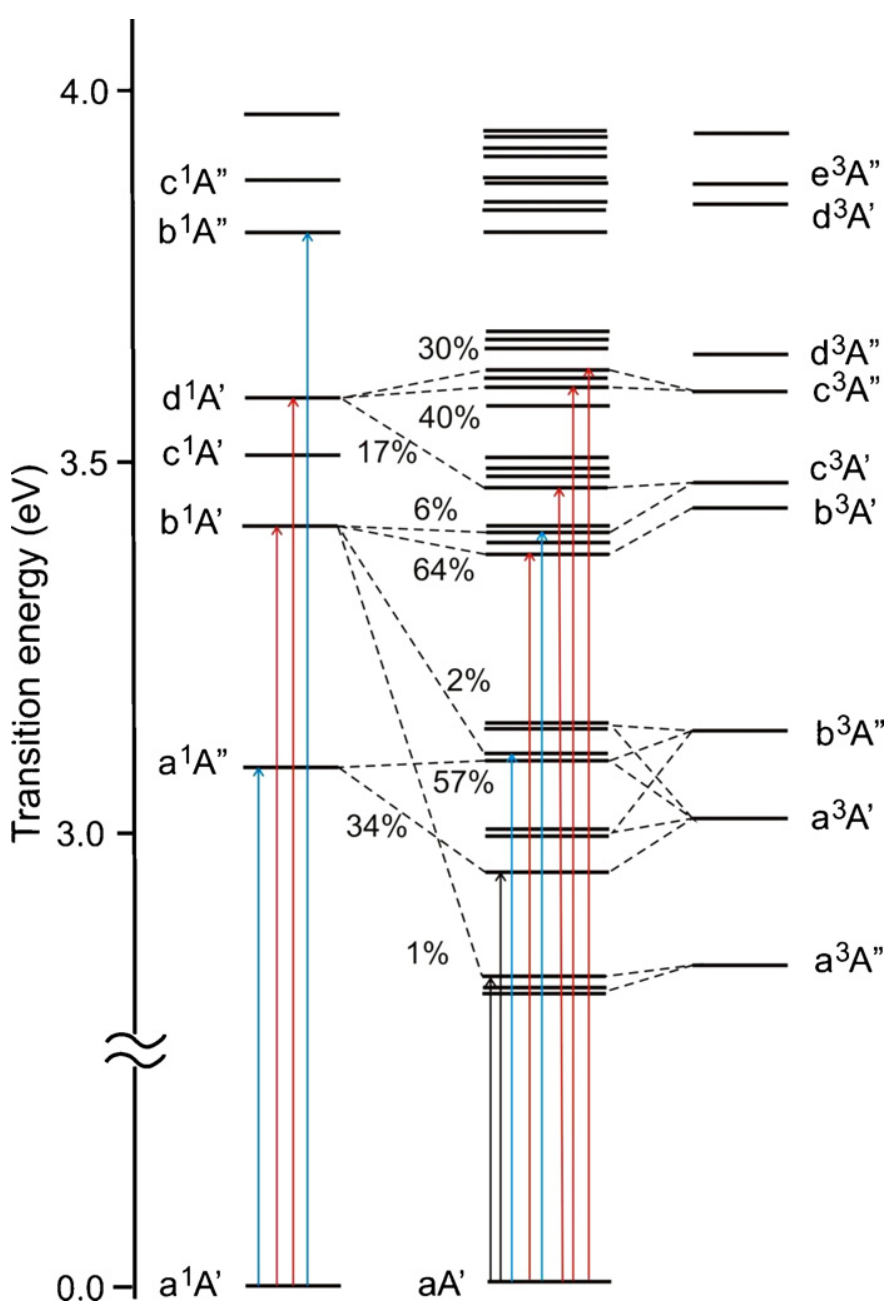

Fig. 3. Correlation of TD-DFT calculated singlet (left) and triplet (right) spin-free states with SO states (middle) of $\left[\operatorname{Re}(\mathrm{imH})(\mathrm{CO})_{3}(\mathrm{phen})\right]^{+}$in DMF solution. Red, blue and black arrows indicate transitions with oscillator strengths larger than 0.01 , 0.001-0.01 and 0.0005-0.001, respectively. SO-TDDFT (PBE0, COSMO-DMF) calculation. Spin-free state characters are as follows: $a^{1} A^{\prime \prime}, b^{1} A, d^{1} A^{\prime}:$ MLCT. Higher-lying singlets contain large IL components. $a^{3} A^{\prime \prime}, a^{3} A^{\prime}$ : MLCT with IL contributions, $b^{3} A^{\prime \prime}$, $\mathrm{b}^{3} \mathrm{~A}^{\prime}$ : IL with MLCT contributions. Higher triplets are strongly mixed. (MLCT contains small imH $\rightarrow$ phen LLCT contributions.)

between metal $\mathrm{d} \pi$ and ligand (L) p $\pi$ orbitals is well described (sometimes even exaggerated) by DFT with hybrid functionals $[8,9,13,62]$, which calculates the lowest two MLCT/LLCT transitions as occurring from occupied $(\mathrm{d} \pi-\mathrm{p} \pi)$ Kohn-Sham molecular orbitals, which are $\pi$-antibonding with respect to the $\mathrm{M}-\mathrm{L}$ bond, to a $\pi^{*}\left(\mathrm{~N}^{\wedge} \mathrm{N}\right)$ orbital. Corresponding two transitions from the $(\mathrm{d} \pi+\mathrm{p} \pi) \pi$-bonding orbitals lie at higher energies depending on $\mathrm{L}$. (Note that $\mathrm{d} \pi$ orbitals are, in addition, mixed with $\pi^{*}(\mathrm{CO})$.) The $\mathrm{d}(\mathrm{Re}), \pi^{*}(\mathrm{CO})$ and $\pi(\mathrm{L})$ contributions are quantified by the respective LCAO coefficients in the KS wavefunction.

(ii) MLCT-IL interconfigurational mixing mostly occurs in triplet states and its extent sensitively depends on the molecular structure and environment [21,22]. It affects the properties of low-lying excited states by diminishing the amount of charge transferred from the metal upon excitation.

TDDFT describes the MLCT-IL mixing in a natural way [9] as a combination of $\mathrm{d} \rightarrow \pi^{*}$ and $\pi \rightarrow \pi^{*}$ one-electron excitations. Electronic transition to any given excited state $E S_{m}$ is represented by a linear combination of one-electron excitations between pairs of 
ground-state KS molecular orbitals $\phi_{p}, \phi_{q}$ :

$\mathrm{GS} \rightarrow \mathrm{ES}_{m}=\sum_{p<q} c_{p q, m}\left(\phi_{p} \rightarrow \phi_{q}\right)$

The square of the expansion coefficient $c_{p q, m}^{2}$ specifies the contribution of the one-electron excitation from the orbital $\phi_{p}$ to the virtual orbital $\phi_{q}$, expressed as a fraction of 1 (or in \%), $\sum_{p<q} c_{p q, m}^{2}=1$ (100). For example, in the particular case of transition to a mixed CT/IL state, we can write:

$\mathrm{GS} \rightarrow \mathrm{CT} / \mathrm{IL}=c_{\mathrm{d} \pi^{*}}\left(\mathrm{~d} \pi \rightarrow \pi^{*}\right)+c_{\pi \pi^{*}}\left(\pi \rightarrow \pi^{*}\right)$

where $\pi$ and $\pi^{*}$ are $\mathrm{N}^{\wedge} \mathrm{N}$-localized orbitals and $\mathrm{d} \pi$ consists of $\mathrm{d}(\mathrm{Re})$, $\pi^{*}(\mathrm{CO})$ and $\pi(\mathrm{L})$. TDDFT-calculated MLCT-IL mixing in the lowest triplet state of $\mathrm{Re}^{\mathrm{I}}$ carbonyl-diimines depends on the dielectric model used to describe the solvent. Experimentally, TRIR spectroscopy shows strong effects of the molecular environment on the amount of the MLCT contribution [15,51,63-66].

Spin-free DFT and TDDFT calculations have been performed on $\left[\operatorname{Re}(\mathrm{X})(\mathrm{CO})_{3}\left(\mathrm{~N}^{\wedge} \mathrm{N}\right)\right] \quad(\mathrm{X}=$ halide $) \quad[9,10,14,15,61]$, $\left[\operatorname{Re}(\mathrm{Etpy})(\mathrm{CO})_{3}(\mathrm{bpy})\right]^{+}$in $\mathrm{MeCN}[10,63],\left[\operatorname{Re}(\mathrm{Et}-\mathrm{imH})(\mathrm{CO})_{3}(\text { phen })\right]^{+}$ in $\mathrm{MeCN}$ [51], $\operatorname{Re}$ (histidine)(CO) ${ }_{3}(\text { phen })^{+}$in an azurin fragment in water [11], and the series $\left[\operatorname{Re}(\mathrm{imH})(\mathrm{CO})_{3}\left(\mathrm{~N}^{\wedge} \mathrm{N}\right)\right]^{+}\left(\mathrm{N}^{\wedge} \mathrm{N}=\right.$ bpy, phen, dmp) in DMF [14]. The lowest $a^{1} A^{\prime} \rightarrow a^{1} A^{\prime \prime}$ MLCT transition due to $\mathrm{HOMO} \rightarrow$ LUMO excitation has a very small oscillator strength because of a poor orbital overlap and is not supposed to contribute significantly to the absorption spectra. The first two allowed transitions $\mathrm{a}^{1} \mathrm{~A}^{\prime} \rightarrow \mathrm{b}, \mathrm{d}^{1} \mathrm{~A}^{\prime}$, which give rise to the lowest absorption band around $360 \mathrm{~nm}$, are composed of HOMO-1,HOMO-2 $\rightarrow$ LUMO excitations in the case of bpy and 80\% HOMO-1,HOMO- $\rightarrow$ LUMO plus $20 \% \mathrm{HOMO} \rightarrow \mathrm{LUMO}+1$ for phen and dmp. These transitions are predominantly $\operatorname{Re}(\mathrm{CO})_{3} \rightarrow \mathrm{N}^{\wedge} \mathrm{N}$ MLCT in character, with a small $\mathrm{imH} \rightarrow \mathrm{N}^{\wedge} \mathrm{N}$ LLCT admixture [14]. Intraligand $\pi \pi^{*}$ transitions follow at higher energies producing intense UV bands $(\sim 320 \mathrm{~nm}$ (bpy), 270-280 $\mathrm{nm}$ (phen, dmp). (The notation used herein assumes the $C_{s}$ symmetry, the letters $a, b, \ldots$ denote the order of states of given spin and symmetry.) Four spin-free triplet states were calculated to occur in a narrow range of about $0.7 \mathrm{eV}$. They are all of a mixed MLCT/IL character. The MLCT component prevails in the lowest triplet state $\mathrm{a}^{3} \mathrm{~A}^{\prime \prime}$, together with a small $\mathrm{imH} \rightarrow \mathrm{N}^{\wedge} \mathrm{N}$ LLCT contribution [14]. The characters of $\left[\operatorname{Re}(\mathrm{imH})(\mathrm{CO})_{3}(\text { phen })\right]^{+}$ spin-free states are summarized in the legend to Fig. 3. (Small differences from the results presented in [14] are caused by different basis sets and solvent models used.)

\section{Spin-orbit excited-state model of $\left[\operatorname{Re}(\mathrm{imH})(\mathrm{CO})_{3}(\mathrm{phen})\right]^{+}$}

\subsection{Theory and techniques}

Spin-orbit coupling (SOC) originates from the interaction between the magnetic moments generated by spin and orbital electron motions [5,67-69]. It couples spatial and spin electron movements, mixes states of different spin multiplicities $(\Delta S=0$, \pm 1 ) and lifts the spin degeneracy of $S$ multiplets by splitting their $M_{S}$ sublevels. Each triplet state thus gives rise to three SO states. $S$ and $M_{\mathrm{S}}$ are no longer "good" quantum numbers. SOC emerges naturally from the Dirac equation, whereas an additional SO Hamiltonian, $\hat{H}_{\text {So, }}$, has to be added into the Schrödinger equation. For polyatomic molecules, $\hat{H}_{\mathrm{SO}}$ is, in principle, a multicenter operator containing one- and two-electron terms:

$\hat{H}_{\text {SO }}=\sum_{A} \sum_{i} \xi\left(r_{i}\right) \boldsymbol{l}_{i}^{A} \cdot \boldsymbol{s}_{i}+2$-electron terms

where $\boldsymbol{l}_{i}^{A}$ is the $i$ th electron angular momentum with respect to the atom $A$ and $s_{i}$ is the spin momentum. $\xi\left(r_{i A}\right)$ is a function of the electron distance from the nucleus $A$ that increases with the atomic number:

$\xi\left(r_{i A}\right)=\frac{\alpha^{2}}{2} \frac{Z_{A}^{e f f}}{\left|\boldsymbol{R}_{A}-\boldsymbol{r}_{i}\right|^{3}}$

$Z_{A}^{e f f}$ stands for an effective nuclear charge, $\alpha$ is the hyperfine splitting constant ( $\sim 1 / 137$ a.u.), and $\boldsymbol{r}_{i}$ is the electron distance from the nucleus $A$ at the position $\boldsymbol{R}_{A}$. (Boldface symbols correspond to 3-dimensional vectors.) The one-electron term describes the interaction between angular and spin magnetic moments of the same electron $(i)$ under the influence of nuclear charges of all atoms $A$ in the molecule. Two-electron terms describe the coupling between the spin momentum of the electron $i$ with the orbital momentum of another electron $(j)$ (and vice versa), and between the electron $i$ and the magnetic moment induced by its movement in the field of the electron $j$ [5]. They provide a partial shielding of the one-electron term that makes a dominant contribution to Eq. (2). Neglecting the 2-electron terms therefore tends to overestimate SOC [5]. SOC rapidly increases with the atomic number because the one-electron term grows with the nuclear charge much faster than the twoelectron ones [5].

Computational implementations of SOC in CASSCF-type and DFT methods have been reviewed recently [5]. In the present work, we have used two different approaches to calculate spin-orbit states of $\left[\operatorname{Re}(\mathrm{imH})(\mathrm{CO})_{3}\left(\mathrm{~N}^{\wedge} \mathrm{N}\right)\right]^{+}$:

(i) Restricted Active Space State Interaction including SOC (RASSISO) within multistate complete active space perturbation theory (CASSCF MS-CASPT2) method incorporated in MOLCAS version 7.4 [70]. RASSI-SO computes matrix elements over spin-orbit states based on first order MS-CASPT2 wave functions [71] using atomic mean-field integrals (AMFI) [72] to account for the 2-electron terms. These calculations were performed in vacuum.

(ii) Approximate SO-TDDFT [73] using Amsterdam Density Functional (ADF) [74] program package. This technique calculates first the sets of spin-free singlet and triplet states and considers SO effects afterwards, producing the double-group symmetry SO states. The solvent was incorporated using the dielectric continuum model COSMO [75-77]. Counter-ion effects were neglected since published electrochemical studies do not indicate any ion-pairing of cationic Re carbonyl-diimines in polar solvents. A test calculation on a small number of states was performed using the computationally much more demanding two-component relativistic TDDFT [78]. The results were comparable with those of the approximate SO-TDDFT [73,79].

Each of these techniques produces a manifold of SO states that are characterized by their energies relative to the ground state, double-group symmetries, parentage in terms of contributing spin-free states, as well as oscillator strengths and Einstein coefficients of spontaneous emission describing the absorption and emission intensities, respectively. The $C_{S}$ symmetry of $\left[\operatorname{Re}(\mathrm{imH})(\mathrm{CO})_{3}(\text { phen })\right]^{+}$was fixed in all calculations.

\subsection{Spin-orbit states at the ground-state geometry: Franck-Condon transitions}

Results of SO calculations of $\left[\operatorname{Re}(\mathrm{imH})(\mathrm{CO})_{3}(\text { phen })\right]^{+}$at the DFToptimized ground-state geometry are summarized in Figs. 2 and 3. Left and right columns show spin-free singlet and triplet states, respectively. SO states are presented in the middle, with dashed lines indicating the principal contributions. SO-state energies, characters and oscillator strengths of transitions from the ground state are summarized in Table 1. SO-state diagrams calculated by RASSI- 
Table 1

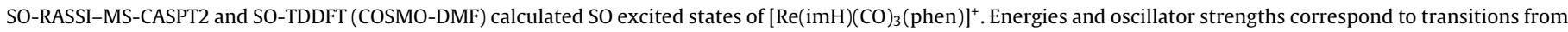
the $\mathrm{aA}^{\prime}$ ground $\mathrm{SO}$ state.

\begin{tabular}{|c|c|c|c|c|c|}
\hline & SO state & Contribution of spin-free states (\%) & Energy $(\mathrm{eV})$ & Energy $\left(\mathrm{cm}^{-1}\right)$ & Osc. strength \\
\hline \multicolumn{6}{|c|}{ SO-RASSI-MS-CASPT2 } \\
\hline 1 & $\mathrm{aA}^{\prime \prime}$ & $a^{3} A^{\prime \prime}(78)+a^{3} A^{\prime}(14)+b^{1} A^{\prime}(1)+c^{3} A^{\prime}(3)$ & 3.166 & 25,535 & 0.0000 \\
\hline 2 & $\mathrm{bA}^{\prime}$ & $a^{3} A^{\prime \prime}(82)+a^{3} A^{\prime}(15)+c^{3} A^{\prime}(1)$ & 3.179 & 25,640 & 0.0000 \\
\hline 3 & $\mathrm{cA}^{\prime}$ & $a^{3} A^{\prime \prime}(92)+b^{1} A^{\prime}(4)+c^{3} A^{\prime}(1)$ & 3.190 & 25,729 & 0.0060 \\
\hline 4 & $\mathrm{bA}^{\prime \prime}$ & $a^{3} A^{\prime}(50)+a^{1} A^{\prime \prime}(46)+c^{3} A^{\prime}(2)+b^{3} A^{\prime}(1)$ & 3.266 & 26,342 & 0.0019 \\
\hline 5 & $\mathrm{cA}^{\prime \prime}$ & $a^{3} A^{\prime}(82)+a^{3} A^{\prime \prime}(17)$ & 3.370 & 27,180 & 0.0000 \\
\hline 6 & $\mathrm{dA}^{\prime \prime}$ & $a^{3} A^{\prime}(84)+a^{3} A^{\prime \prime}(15)$ & 3.370 & 27,181 & 0.0000 \\
\hline 7 & $\mathrm{dA}^{\prime}$ & $a^{3} A^{\prime}(49)+a^{1} A^{\prime \prime}(49)+b^{3} A^{\prime}(1)$ & 3.428 & 27,649 & 0.0024 \\
\hline 8 & $\mathrm{eA}^{\prime}$ & $b^{1} A^{\prime}(84)+a^{3} A^{\prime \prime}(7)+b^{3} A^{\prime \prime}(5)$ & 3.591 & 28,963 & 0.0437 \\
\hline 9 & $\mathrm{fA}^{\prime}$ & $c^{1} A^{\prime}(74)+b^{3} A^{\prime \prime}(15)+b^{3} A^{\prime}(4)+a^{3} A^{\prime \prime}(3)+a^{3} A^{\prime}(2)$ & 3.709 & 29,915 & 0.0364 \\
\hline 10 & $\mathrm{eA}^{\prime \prime}$ & $b^{3} A^{\prime \prime}(87)+b^{3} A^{\prime}(6)+c^{3} A^{\prime}(5)$ & 3.824 & 30,843 & 0.0000 \\
\hline 11 & $\mathrm{fA}^{\prime \prime}$ & $b^{3} A^{\prime \prime}(63)+d^{1} A^{\prime}(26)+b^{1} A^{\prime}(5)$ & 3.840 & 30,972 & 0.0010 \\
\hline 12 & $\mathrm{gA}^{\prime}$ & $b^{3} A^{\prime \prime}(72)+c^{1} A^{\prime}(15)+d^{1} A^{\prime}(10)$ & 3.865 & 31,173 & 0.0158 \\
\hline 13 & $\mathrm{hA}^{\prime}$ & $d^{1} A^{\prime}(57)+b^{3} A^{\prime \prime}(36)+c^{3} A^{\prime \prime}(5)$ & 3.880 & 31,294 & 0.0191 \\
\hline \multicolumn{6}{|c|}{ SO-TDDFT } \\
\hline 1 & $\mathrm{aA}^{\prime \prime}$ & $a^{3} A^{\prime \prime}(91)+a^{3} A^{\prime}(6)$ & 2.791 & 22,511 & 0.0000 \\
\hline 2 & $\mathrm{bA}^{\prime}$ & $a^{3} A^{\prime \prime}(91)+a^{3} A^{\prime}(6)+c^{3} A^{\prime}(1)$ & 2.792 & 22,519 & 0.0003 \\
\hline 3 & $\mathrm{cA}^{\prime}$ & $a^{3} A^{\prime \prime}(96)+b^{1} A^{\prime}(1)+b^{3} A^{\prime}(1)$ & 2.799 & 22,575 & 0.0006 \\
\hline 4 & $\mathrm{bA}^{\prime \prime}$ & $a^{3} A^{\prime}(60)+a^{1} A^{\prime \prime}(34)+c^{3} A^{\prime}(2)+b^{3} A^{\prime}(2)$ & 2.945 & 23,753 & 0.0005 \\
\hline 5 & $\mathrm{cA}^{\prime \prime}$ & $a^{3} A^{\prime}(82)+a^{3} A^{\prime \prime}(17)$ & 2.992 & 24,132 & 0.0000 \\
\hline 6 & $\mathrm{dA}^{\prime}$ & $a^{3} A^{\prime}(76)+a^{3} A^{\prime \prime}(7)+b^{3} A^{\prime \prime}(15)$ & 2.992 & 24,132 & 0.0002 \\
\hline 7 & $\mathrm{dA}^{\prime \prime}$ & $a^{1} A^{\prime \prime}(57)+a^{3} A^{\prime}(38)$ & 3.104 & 25,035 & 0.0004 \\
\hline 8 & $\mathrm{eA}^{\prime}$ & $b^{3} A^{\prime \prime}(91)+c^{3} A^{\prime}(3)+b^{1} A^{\prime}(2)$ & 3.106 & 25,052 & 0.0017 \\
\hline 9 & $\mathrm{eA}^{\prime \prime}$ & $b^{3} A^{\prime \prime}(79)+a^{3} A^{\prime}(18)$ & 3.139 & 25,318 & 0.0000 \\
\hline 10 & $\mathrm{fA}^{\prime}$ & $b^{3} A^{\prime \prime}(80)+a^{3} A^{\prime}(17)$ & 3.141 & 25,334 & 0.0003 \\
\hline 11 & $\mathrm{gA}^{\prime}$ & $b^{1} A^{\prime}(64)+b^{3} A^{\prime}(14)+c^{3} A^{\prime}(5)+c^{1} A^{\prime}(4)+c^{3} A^{\prime \prime}(4)$ & 3.381 & 27,270 & 0.0219 \\
\hline 12 & $\mathrm{fA}^{\prime \prime}$ & $b^{3} A^{\prime}(67)+c^{3} A^{\prime}(20)+d^{3} A^{\prime}(5)$ & 3.392 & 27,358 & 0.0000 \\
\hline 13 & $\mathrm{hA}^{\prime}$ & $b^{3} A^{\prime}(57)+c^{3} A^{\prime}(23)+b^{1} A^{\prime}(6)$ & 3.395 & 27,382 & 0.0026 \\
\hline 14 & $\mathrm{gA}^{\prime \prime}$ & $b^{3} A^{\prime}(62)+c^{3} A^{\prime}(25)+b^{1} A^{\prime \prime}(5)$ & 3.397 & 27,399 & 0.0009 \\
\hline 15 & $\mathrm{iA}^{\prime}$ & $c^{3} A^{\prime}(48)+b^{3} A^{\prime}(11)+d^{1} A^{\prime}(17)+b^{1} A^{\prime}(8)+c^{1} A^{\prime}(5)$ & 3.471 & 27,995 & 0.0173 \\
\hline 16 & $\mathrm{hA}^{\prime \prime}$ & $c^{3} A^{\prime}(63)+b^{3} A^{\prime}(23)+d^{3} A^{\prime}(5)+b^{3} A^{\prime \prime}(8)$ & 3.473 & 28,012 & 0.0000 \\
\hline 17 & $\mathrm{iA}^{\prime \prime}$ & $c^{3} A^{\prime}(65)+b^{3} A^{\prime}(21)+b^{1} A^{\prime \prime}(6)$ & 3.476 & 28,036 & 0.0002 \\
\hline 18 & $\mathrm{j} \mathrm{A}^{\prime}$ & $c^{1} A^{\prime}(67)+d^{3} A^{\prime}(8)+c^{3} A^{\prime}(7)+b^{1} A^{\prime}(4)$ & 3.500 & 28,229 & 0.0042 \\
\hline 19 & $\mathrm{kA}^{\prime}$ & $d^{1} A^{\prime}(40)+d^{3} A^{\prime}(38)+b^{3} A^{\prime}(5)+b^{1} A^{\prime}(5)$ & 3.579 & 28,867 & 0.0361 \\
\hline 20 & $\mathrm{j} \mathrm{A}^{\prime \prime}$ & $c^{3} A^{\prime \prime}(93)$ & 3.596 & 29,004 & 0.0000 \\
\hline 21 & $1 A^{\prime}$ & $d^{1} A^{\prime}(30)+c^{3} A^{\prime \prime}(58)+c^{3} A^{\prime}(6)+c^{1} A^{\prime}(3)$ & 3.605 & 29,076 & 0.0251 \\
\hline
\end{tabular}

SO/MS-CASPT2 (Fig. 2) and SO-TDDFT (Fig. 3) methods show similar qualitative features for the lowest six SO states; the small differences occurring at higher energies stem from the much larger gap between the spin-free $a^{3} \mathrm{~A}^{\prime}$ and $\mathrm{b}^{3} \mathrm{~A}^{\prime \prime}$ states in MS-CASPT2 than TDDFT. The following discussion will be based on the results obtained with the SO-TDDFT technique [73] that is computationally feasible and, at least partly, accounts for the solvent effects.

The three lowest $\mathrm{SO}$ states have $\mathrm{A}^{\prime \prime}, \mathrm{A}^{\prime}$ and $\mathrm{A}^{\prime}$ symmetries, denoted $a A^{\prime \prime}, b^{\prime}$ and $c A^{\prime}$ in the order of increasing energy. They are predominantly $a^{3} A^{\prime \prime}$ MLCT in origin. The lowest $a A^{\prime \prime}$ state is spectroscopically forbidden whereas transitions to the second and third states $\left(\mathrm{bA}^{\prime}, \mathrm{cA}^{\prime}\right)$ have very small oscillator strengths and could weakly contribute to the red tail of the absorption band. The overall separation of the lowest three states $\mathrm{CA}^{\prime}-\mathrm{aA}^{\prime \prime}$ was calculated as $64 \mathrm{~cm}^{-1}$ by SO-TDDFT and $194 \mathrm{~cm}^{-1}$ by CASPT2. The first weakly allowed transition occurs to the mixed MLCT-IL state $\mathrm{eA}^{\prime}$ that consists of $94 \%$ triplet character, gaining intensity through the $2 \%$ $b^{1} A^{\prime}$ contribution. The first fully allowed transition is directed to $\mathrm{gA}^{\prime}$ MLCT with a small IL admixture, followed by transitions to $\mathrm{hA}^{\prime}$ and $\mathrm{iA}^{\prime \prime}$. Fig. 3 shows that there are more allowed (or semiallowed) transitions to SO states than to spin-free singlets. The higher density of SO states thus accounts well for the broad and asymmetric shape of the experimental lowest absorption band of $\left[\operatorname{Re}(\mathrm{imH})(\mathrm{CO})_{3}(\text { phen })\right]^{+}$that extends from $\mathrm{ca} .320$ to $420 \mathrm{~nm}$ [14]. The effect of SOC on electronic transitions and simulated absorption spectrum is shown in Fig. 4. Although the absorption bands calculated with and without SOC are very similar, their origin is quite different: the slightly weaker and broader SO absorption band arises from a large number of weaker transitions whereas only

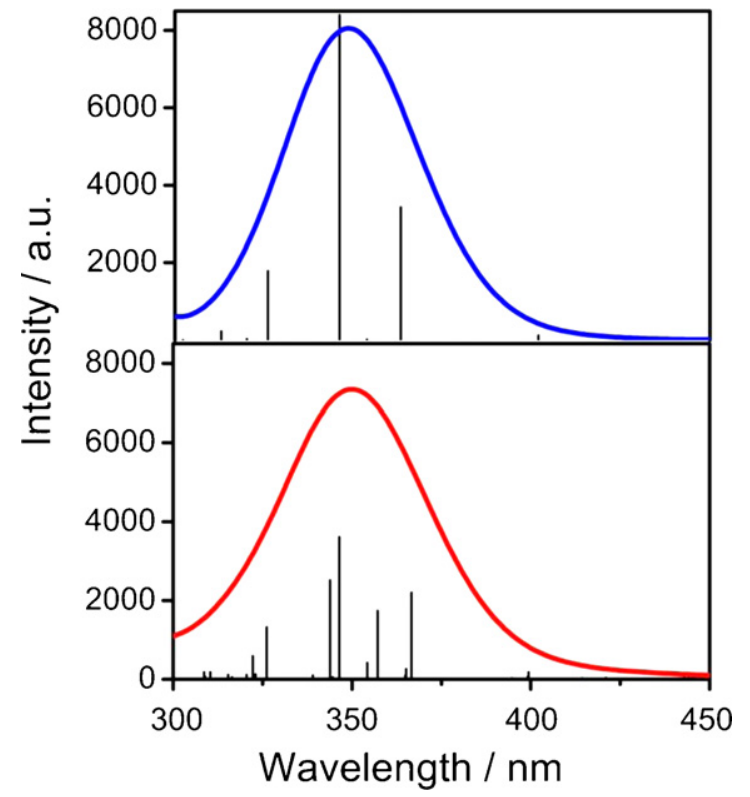

Fig. 4. Spin-free TDDFT (top) and SO-TDDFT (bottom) simulated absorption spectra and electronic transitions of $\left[\operatorname{Re}(\mathrm{imH})(\mathrm{CO})_{3}(\mathrm{phen})\right]^{+}$in DMF. (Simulated with Gaussian band-shapes assuming $3000 \mathrm{~cm}^{-1}$ fwhm.) The experimental spectrum [14] shows a broad absorption with distinct shoulders at ca. 362 and $328 \mathrm{~nm}$, followed by a strong absorption at shorter wavelengths, due to transitions not included in the present simulation. 
three strong transitions make for the spin-free band. It is important to note that even the spectroscopically allowed SO states contain significant triplet components, e.g. 23\% for gA'. Moreover, the SO states are much more mixed than the spin-free ones in terms of their character (i.e. orbital parentage), making it difficult to describe them in terms of MLCT, LLCT or IL excitations.

\subsection{Spin-orbit states at excited-state geometries: towards interpreting photophysics}

Photophysics and excited-state dynamics of $\operatorname{Re}^{\mathrm{I}}$ carbonyl diimines have been studied in detail $[10,14,15,22,24,80]$. The following salient experimental observations were made on $\left[\operatorname{Re}(\mathrm{imH})(\mathrm{CO})_{3}(\text { phen })\right]^{+}$, which are typical for many other $\operatorname{Re}^{\mathrm{I}}$ carbonyl-diimines complexes:

1. Femtosecond laser pulse excitation at $400 \mathrm{~nm}\left(25,000 \mathrm{~cm}^{-1}\right)$ triggers emission ("fluorescence") characterized by a very broad band at $\sim 530 \mathrm{~nm}$ that emerges within the instrument time resolution, i.e. faster than $30 \mathrm{fs}$.

2. Fluorescence decays with a $\sim 150$ fs lifetime with a simultaneous population of two states: an intermediate one that shows a weak broad emission ( $\sim 580 \mathrm{~nm})$ and decays with a 1.5 ps lifetime, and the lowest excited state that gives rise to a broad emission at $\sim 590 \mathrm{~nm}$, which decays with a 120 ns lifetime [44].

3. Time-resolved UV-vis spectroscopy reveals further relaxation processes occurring with time-constants $2.0,3.9,14$, and $28 \mathrm{ps,}$ manifested by small intensity- and band-shape changes. Another 8.5 ps relaxation is revealed by dynamic IR spectroscopic shifts [80].

4. TRIR spectra indicate the presence of two long-lived equilibrated excited states [14]. The "major" state has a predominantly MLCT character. The "minor" state (10-20\% population) is MLCT with a substantial IL admixture. Both states seem to be populated simultaneously after optical excitation, establishing a thermal equilibrium within a few ps [14].

5. The lowest excited state(s) of $\operatorname{Re}^{\mathrm{I}}$ carbonyl-diimines $\left[\operatorname{Re}(\mathrm{L})(\mathrm{CO})_{3}\left(\mathrm{~N}^{\wedge} \mathrm{N}\right)\right]^{\mathrm{n}}$ typically show a temperaturedependent emission decay, with activation energy in the range $120-1430 \mathrm{~cm}^{-1}$, depending on the diimine and $\mathrm{L}[81,82]$. (Temperature-dependent lifetime data for $\left[\operatorname{Re}(\mathrm{imH})(\mathrm{CO})_{3}(\text { phen })\right]^{+}$are not available.)

The observed excited-state behavior has been interpreted $[10,14,15,22]$ within the spin-free conceptual framework, as depicted in Fig. 5: Laser-pulse excitation of the lowest allowed transition of $\left[\operatorname{Re}(\mathrm{imH})(\mathrm{CO})_{3}(\text { phen })\right]^{+}$populates the fluorescent $\mathrm{b}^{1} \mathrm{~A}^{\prime}$ MLCT state. Excitation is immediately followed by vibrational energy redistribution manifested by the "instantaneous" fluorescence Stokes shift. The $\mathrm{b}^{1} \mathrm{~A}^{\prime}$ state undergoes $\sim 150 \mathrm{fs}$ intersystem crossing to the low-lying, long-lived phosphorescent MLCT state $a^{3} A^{\prime \prime}$. Another "intermediate" phosphorescent state, presumably $b^{3} \mathrm{~A}^{\prime \prime}$, is populated on the way and decays fast to $\mathrm{a}^{3} \mathrm{~A}^{\prime \prime}$. Vibrational and solvational relaxations of $\mathrm{b}^{3} \mathrm{~A}^{\prime \prime}$ and $\mathrm{a}^{3} \mathrm{~A}^{\prime \prime}$ states also contribute to the observed excited-state dynamics. The spin-free approach provides a relatively simple photophysical model that explains the principal experimental observations, although it has problems with the presence of different relaxation times observed in absorption and emission, the presence of the second low-lying triplet state detected by TRIR, and explaining the temperature dependence of emission lifetimes.

Next, we will examine the power of the SO model in interpreting $\left[\operatorname{Re}(\mathrm{imH})(\mathrm{CO})_{3}(\text { phen })\right]^{+}$photophysics. A rigorous treatment would require calculate potential energy surfaces along SO-state decay pathways and examine the state crossing regions. Since this task is beyond current computational means, we have calcu-

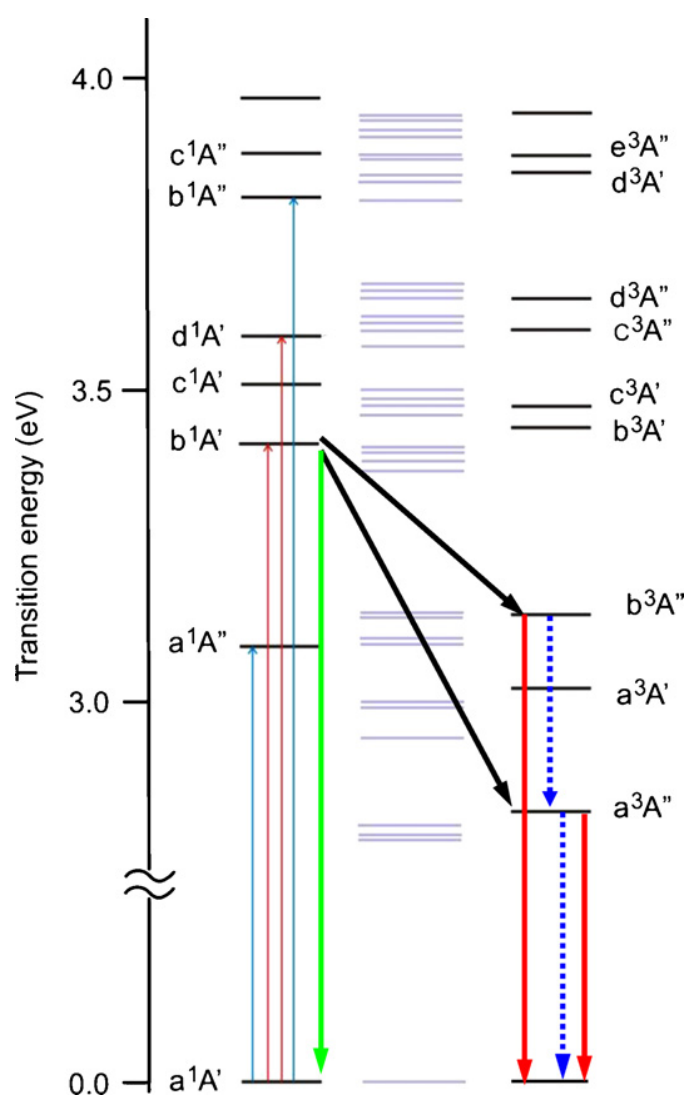

Fig. 5. Spin-free photophysical model of $\left[\operatorname{Re}(\mathrm{imH})(\mathrm{CO})_{3}(\text { phen })\right]^{+}$and other $\operatorname{Re}^{\mathrm{I}}$ carbonyl-diimine complexes $[10,14,15,22]$. Optical excitation populates the $\mathrm{b}^{1} \mathrm{~A}^{\prime}$ state that undergoes fluorescence (green arrow) and simultaneous ISC to $\mathrm{b}^{3} \mathrm{~A}^{\prime \prime}$ and $\mathrm{a}^{3} \mathrm{~A}^{\prime \prime}$ (black arrows). Phosphorescence is shown by red arrows. The blue dotted arrows depict nonradiative decays of the two triplet states. Note, that the very occurrence of ISC, phosphorescence and $\mathrm{a}^{3} \mathrm{~A}^{\prime \prime} \rightarrow \mathrm{a}^{1} \mathrm{~A}^{\prime}$ nonradiative decay implicitly requires SOC. SO states are indicated in the middle column, but not considered in the spin-free model. Vibrational relaxation of the electronic states involved is omitted for clarity.

lated the SO states at the geometries (Table A1 - Appendix A) of the $a^{1} A^{\prime}$ ground state and of the relaxed $b^{1} A^{\prime}$ and $a^{3} A^{\prime \prime}$ spin-free excited states, Fig. 6 and Table 2. The latter two approximate the structures of the optically populated SO state(s) and of the lowest three SO states, respectively. This approximation is used here to estimate effects of the structural changes during excited-state evolution on the whole SO-state manifold and indicate a possible approach to developing SO photophysical and photochemical models.

In the SO model (Figs. 6 and 7), optical excitation at $400 \mathrm{~nm}$ populates predominantly the gA' MLCT state, together with MLCT-IL states $\mathrm{iA}^{\prime}, \mathrm{hA}^{\prime}$, and $\mathrm{eA}^{\prime}$. The $g \mathrm{~A}^{\prime}$ state has a $\sim 23 \%$ triplet parentage. Changing the geometry to that of the spin-free $b^{1} A^{\prime}$ state simulates the effect of the observed ultrafast ( $<30 \mathrm{fs}$ ) intramolecular vibrational redistribution. It stabilizes all the SO states except $\mathrm{eA}^{\prime}$ and increases the energy difference between the third and the lowest two SO states, which both acquire about $22 \% \mathrm{a}^{3} \mathrm{~A}^{\prime}$ at the expense of the $a^{3} A^{\prime \prime}$ content. A group of potentially strongly emissive states $\left(\mathrm{eA}^{\prime}, \mathrm{fA}^{\prime}, \mathrm{gA}^{\prime}, \mathrm{hA^{ \prime }}, \mathrm{iA^{ \prime }}, \mathrm{hA}^{\prime \prime}, \mathrm{jA}^{\prime}, \mathrm{kA}^{\prime}\right)$ is calculated in the region of $23,117-26,640 \mathrm{~cm}^{-1}$, below the Franck-Condon $\mathrm{gA}^{\prime}$ excitation energy of $27,270 \mathrm{~cm}^{-1}$. The $\mathrm{gA}^{\prime}$ state itself loses a lot of its $\mathrm{b}^{1} \mathrm{~A}^{\prime}$ character and becomes much more triplet-like due to increasing $\mathrm{b}^{3} \mathrm{~A}^{\prime \prime}$ and $\mathrm{c}^{3} \mathrm{~A}^{\prime}$ contributions. Interesting is also the $\mathrm{eA}^{\prime}$ state that shifts to higher energy, acquires additional $43 \%$ singlet character and becomes spectroscopically much more allowed. 
Table 2

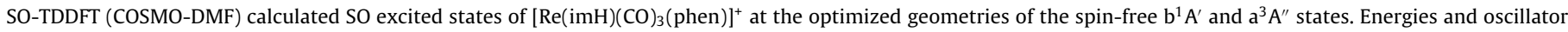
strengths correspond to transitions from the $\mathrm{aA}^{\prime}$ ground SO state at given geometry. The last column shows the Einstein spontaneous emission coefficient $A$.

\begin{tabular}{|c|c|c|c|c|c|c|}
\hline & SO state & Contribution of spin-free states (\%) & Energy $(\mathrm{eV})$ & Energy $\left(\mathrm{cm}^{-1}\right)$ & Oscillator strength & Emiss. coeff. $\left(10^{7} \mathrm{~s}^{-1}\right)$ \\
\hline \multicolumn{7}{|c|}{$b^{1} A^{\prime}$ geometry } \\
\hline 1 & $\mathrm{aA}^{\prime \prime}$ & $a^{3} A^{\prime \prime}(68)+a^{3} A^{\prime}(28)+b^{3} A^{\prime}(2)$ & 2.368 & 19,102 & 0.0000 & 0.000 \\
\hline 2 & $\mathrm{bA}^{\prime}$ & $a^{3} A^{\prime \prime}(68)+a^{3} A^{\prime}(28)+c^{1} A^{\prime}(2)$ & 2.370 & 19,112 & 0.0010 & 0.024 \\
\hline 3 & $\mathrm{cA}^{\prime}$ & $a^{3} A^{\prime \prime}(94)+b^{1} A^{\prime}(3)+c^{3} A^{\prime}(2)$ & 2.407 & 19,417 & 0.0022 & 0.055 \\
\hline 4 & $\mathrm{bA}^{\prime \prime}$ & $a^{3} A^{\prime}(78)+a^{1} A^{\prime \prime}(19)+b^{3} A^{\prime}(3)$ & 2.450 & 19,761 & 0.0002 & 0.005 \\
\hline 5 & $\mathrm{cA}^{\prime \prime}$ & $a^{3} A^{\prime}(68)+a^{3} A^{\prime \prime}(30)$ & 2.545 & 20,528 & 0.0000 & 0.000 \\
\hline 6 & $\mathrm{dA}^{\prime}$ & $a^{3} A^{\prime}(68)+a^{3} A^{\prime \prime}(30)$ & 2.545 & 20,529 & 0.0001 & 0.003 \\
\hline 7 & $\mathrm{dA}^{\prime \prime}$ & $a^{1} A^{\prime \prime}(76)+a^{3} A^{\prime}(21)$ & 2.694 & 21,730 & 0.0006 & 0.019 \\
\hline 8 & $\mathrm{eA}^{\prime}$ & $b^{1} A^{\prime}(45)+b^{3} A^{\prime \prime}(36)+b^{3} A^{\prime}(13)$ & 2.866 & 23,117 & 0.0308 & 1.098 \\
\hline 9 & $\mathrm{eA}^{\prime \prime}$ & $b^{3} A^{\prime \prime}(88)+b^{3} A^{\prime}(7)$ & 2.924 & 23,579 & 0.0000 & 0.000 \\
\hline 10 & $\mathrm{fA}^{\prime}$ & $b^{3} A^{\prime \prime}(90)+b^{3} A^{\prime}(6)+b^{1} A^{\prime}(2)$ & 2.925 & 23,593 & 0.0019 & 0.071 \\
\hline 11 & $\mathrm{gA}^{\prime}$ & $b^{1} A^{\prime}(34)+b^{3} A^{\prime \prime}(60)+c^{1} A^{\prime}(1)$ & 2.959 & 23,862 & 0.0242 & 0.919 \\
\hline 12 & $\mathrm{fA}^{\prime \prime}$ & $b^{3} A^{\prime}(84)+c^{3} A^{\prime \prime}(6)+c^{3} A^{\prime}(4)$ & 3.040 & 24,519 & 0.0000 & 0.000 \\
\hline 13 & $\mathrm{gA}^{\prime \prime}$ & $b^{3} A^{\prime}(88)+a^{1} A^{\prime \prime}(5)$ & 3.046 & 24,565 & 0.0001 & 0.004 \\
\hline 14 & $\mathrm{hA}^{\prime}$ & $b^{3} A^{\prime}(69)+b^{1} A^{\prime}(12)+c^{1} A^{\prime}(8)$ & 3.049 & 24,589 & 0.0100 & 0.403 \\
\hline 15 & $i A^{\prime}$ & $c^{1} A^{\prime}(77)+b^{3} A^{\prime}(11)+c^{3} A^{\prime}(6)$ & 3.159 & 25,479 & 0.0198 & 0.857 \\
\hline 16 & $\mathrm{hA}^{\prime \prime}$ & $c^{3} A^{\prime}(82)+b^{1} A^{\prime \prime}(10)$ & 3.202 & 25,826 & 0.0019 & 0.085 \\
\hline 17 & $\mathrm{iA}^{\prime \prime}$ & $c^{3} A^{\prime}(76)+b^{3} A^{\prime \prime}(11)$ & 3.212 & 25,910 & 0.0001 & 0.004 \\
\hline 18 & $\mathrm{jA}^{\prime}$ & $c^{3} A^{\prime}(72)+c^{1} A^{\prime}(8)+c^{3} A^{\prime \prime}(10)$ & 3.214 & 25,924 & 0.0029 & 0.130 \\
\hline 19 & $\mathrm{kA}^{\prime}$ & $c^{3} A^{\prime}(68)+d^{1} A^{\prime}(24)$ & 3.303 & 26,640 & 0.0148 & 0.701 \\
\hline \multicolumn{7}{|c|}{$a^{3} A^{\prime \prime}$ geometry } \\
\hline 1 & $\mathrm{aA}^{\prime \prime}$ & $a^{3} A^{\prime \prime}(94)+a^{3} A^{\prime}(3)$ & 2.080 & 16,778 & 0.0000 & 0.000 \\
\hline 2 & $\mathrm{bA}^{\prime}$ & $a^{3} A^{\prime \prime}(94)+a^{3} A^{\prime}(3)+c^{3} A^{\prime}(2)$ & 2.081 & 16,787 & 0.0001 & 0.002 \\
\hline 3 & $\mathrm{cA}^{\prime}$ & $a^{3} A^{\prime \prime}(96)+c^{1} A^{\prime}(1)+c^{3} A^{\prime}(1)$ & 2.088 & 16,845 & 0.0005 & 0.009 \\
\hline 4 & $\mathrm{bA}^{\prime \prime}$ & $a^{1} A^{\prime \prime}(85)+a^{3} A^{\prime}(10)$ & 2.252 & 18,167 & 0.0011 & 0.024 \\
\hline 5 & $\mathrm{cA}^{\prime \prime}$ & $a^{3} A^{\prime}(92)+a^{3} A^{\prime \prime}(4)$ & 2.525 & 20,363 & 0.0000 & 0.000 \\
\hline 6 & $\mathrm{dA}^{\prime}$ & $a^{3} A^{\prime}(93)+a^{3} A^{\prime \prime}(4)$ & 2.525 & 20,369 & 0.0001 & 0.003 \\
\hline 7 & $\mathrm{dA}^{\prime \prime}$ & $a^{3} A^{\prime}(88)+a^{1} A^{\prime \prime}(4)$ & 2.545 & 20,528 & 0.0002 & 0.006 \\
\hline 8 & $\mathrm{eA}^{\prime}$ & $b^{3} A^{\prime}(70)+c^{1} A^{\prime}(13)+b^{3} A^{\prime \prime}(4)$ & 2.827 & 22,802 & 0.0063 & 0.218 \\
\hline 9 & $\mathrm{eA}^{\prime \prime}$ & $b^{3} A^{\prime}(80)+b^{3} A^{\prime \prime}(11)$ & 2.864 & 23,102 & 0.0000 & 0.000 \\
\hline 10 & $\mathrm{fA}^{\prime \prime}$ & $b^{3} A^{\prime}(86)+c^{3} A^{\prime}(8)+a^{1} A^{\prime \prime}(3)$ & 2.879 & 23,219 & 0.0001 & 0.004 \\
\hline 11 & $\mathrm{fA}^{\prime}$ & $b^{1} A^{\prime}(63)+b^{3} A^{\prime \prime}(30)+a^{3} A^{\prime}(3)$ & 2.891 & 23,321 & 0.0015 & 0.054 \\
\hline 12 & $\mathrm{gA}^{\prime \prime}$ & $b^{3} A^{\prime \prime}(48)+c^{3} A^{\prime}(46)$ & 2.913 & 23,492 & 0.0000 & 0.000 \\
\hline 13 & $\mathrm{gA}^{\prime}$ & $c^{3} A^{\prime}(52)+b^{3} A^{\prime \prime}(32)+b^{1} A^{\prime}(11)$ & 2.914 & 23,506 & 0.0003 & 0.011 \\
\hline 14 & $\mathrm{hA}^{\prime}$ & $c^{1} A^{\prime}(35)+b^{3} A^{\prime \prime}(58)+d^{1} A^{\prime}(4)$ & 2.919 & 23,543 & 0.0160 & 0.592 \\
\hline 15 & $\mathrm{hA}^{\prime \prime}$ & $c^{3} A^{\prime}(86)+b^{3} A^{\prime}(8)$ & 2.954 & 23,824 & 0.0002 & 0.008 \\
\hline 16 & $i A^{\prime}$ & $c^{1} A^{\prime}(44)+b^{3} A^{\prime \prime}(28)+b^{3} A^{\prime}(25)$ & 2.979 & 24,026 & 0.0149 & 0.574 \\
\hline 17 & $\mathrm{iA}^{\prime \prime}$ & $b^{3} A^{\prime \prime}(40)+c^{3} A^{\prime}(47)$ & 2.987 & 24,095 & 0.0000 & 0.000 \\
\hline 18 & $\mathrm{jA}^{\prime}$ & $c^{3} A^{\prime}(41)+b^{3} A^{\prime \prime}(37)+b^{1} A^{\prime}(17)$ & 2.993 & 24,139 & 0.0011 & 0.043 \\
\hline
\end{tabular}

On going to the $\mathrm{a}^{3} \mathrm{~A}^{\prime \prime}$ geometry, we see further stabilization of the whole SO-state manifold while the individual SO states tend to cluster into four groups. A set of strongly emissive states occurs at relatively high energies, 22,802-25,576 $\mathrm{cm}^{-1}\left(\mathrm{eA}^{\prime}, \mathrm{fA}^{\prime}, \mathrm{hA} \mathrm{A}^{\prime}, \mathrm{iA}\right.$, $\mathrm{jA}^{\prime}, \mathrm{kA}^{\prime}$ ). The lower-lying $\mathrm{cA}^{\prime \prime}, \mathrm{dA}^{\prime}, \mathrm{dA}^{\prime \prime}$ group of states does not seem to be of much photophysical importance. Interesting is the fourth SO state $\mathrm{bA}^{\prime \prime}$, that is predominantly $\mathrm{a}^{3} \mathrm{~A}^{\prime} \mathrm{MLCT}(60 \%)$ in the ground-state structure, becoming mostly singlet $\left(85 \% \mathrm{a}^{1} \mathrm{~A}^{\prime \prime}\right.$ MLCT) at the $\mathrm{a}^{3} \mathrm{~A}^{\prime \prime}$ geometry. It is 2.7-times more emissive than the third state $\mathrm{CA}^{\prime}$. The lowest three SO states become nearly pure $\mathrm{a}^{3} \mathrm{~A}^{\prime \prime}$ triplets; the third state $\mathrm{cA}^{\prime}$ is weakly emissive due to the $1 \%$ $c^{1} A^{\prime}$ contribution. Its calculated energy, $16,845 \mathrm{~cm}^{-1}$ compares well with that of the emission band maximum at $16,949 \mathrm{~cm}^{-1}(590 \mathrm{~nm})$ [44]. The total ZFS is calculated as $67 \mathrm{~cm}^{-1}$, comparable to the value of $42.1 \mathrm{~cm}^{-1}$ measured $[83,84]$ for $\left[\operatorname{Re}(\mathrm{Cl})(\mathrm{CO})_{3}(\right.$ phen $\left.)\right]$ in a low-temperature matrix. Assuming Boltzman equilibrium between the relaxed excited states, we can calculate the relative populations of the lowest four states as a function of temperature, Table 3. The majority of the excited population is distributed amongst the three lowest states. The $\mathrm{bA}^{\prime \prime}$ thermal population is negligible but it can still provide an efficient thermally activated decay pathway (radiative as well as nonradiative) to the ground state. (Note, that the populations reported in Table 3 are approximate since the same geometry is assumed for the first three SO states and $\mathrm{bA}^{\prime \prime}$. It can be argued that the actual bA" energy at its relaxed geometry is lower, leading to a higher relative population.)
Knowing the SO-state characters and energies, we will now attempt to explain the photophysics in terms of SO states, Fig. 7. Laser-pulse excitation populates predominantly the Franck-Condon gA' $^{\prime}$ state, together with a group of close-lying states I. The following ultrafast relaxation ( $<30 \mathrm{fs}$ ), manifested by ultrafast "instantaneous" fluorescence Stokes shift from the excitation wavelength of $400 \mathrm{~nm}\left(25,000 \mathrm{~cm}^{-1}\right)$ to $\sim 530 \mathrm{~nm}\left(\sim 18,900 \mathrm{~cm}^{-1}\right)$ seems to include both IVR, that will distort the molecular structure towards that approximated by the spin-free $b^{1} \mathrm{~A}^{\prime}$ state, and electronic energy redistribution amongst the densely packed manifold of emissive states II. The prompt "fluorescence" observed at $\sim 530 \mathrm{~nm}$ is proposed to originate from this group of SO states, which are, furthermore, excited vibrationally. This combined multiple electronic and vibrational excitation accounts for the enormous spectroscopic broadness (460-600 nm half-width) of the "fluorescence" band observed for $\left[\operatorname{Re}(\mathrm{imH})(\mathrm{CO})_{3}(\text { phen })\right]^{+}$and other $\operatorname{Re}^{\mathrm{I}}$ carbonyl diimines $[10,14,15]$. Given the large triplet content in the emissive SO states (except for $\mathrm{iA}^{\prime}$ ), it is better to talk about prompt emission than fluorescence. The $\sim 150$ fs emission decay is now attributed to a nonradiative conversion of the group of states II into the lowest four SO states, all vibrationally hot and in a unequilibrated solvent arrangement. It is conceivable that this conversion is facilitated by the increase of the triplet character upon changing the molecular structure of the optically populated states. The initial population distribution of the low-lying SO states will be far from equilibrium, determined kinetically. The intermediate emission is tentatively assigned to the fourth state $\mathrm{bA}^{\prime \prime}$ that decays with 
Table 3

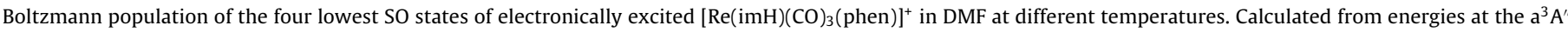
geometries, presented in Table 2. Last two columns: values obtained with $E\left(\mathrm{cA}^{\prime}\right)=0.016 \mathrm{eV}$ and $E\left(\mathrm{bA}^{\prime \prime}\right)=0.066 \mathrm{eV}$ above aA", respectively. All other values as in Table 2 .

\begin{tabular}{|c|c|c|c|c|c|c|}
\hline & $253 \mathrm{~K}$ & $273 \mathrm{~K}$ & $293 \mathrm{~K}$ & $313 \mathrm{~K}$ & $273 \mathrm{~K} E\left(\mathrm{cA}^{\prime}\right)$ doubled & $273 \mathrm{~K} E\left(\mathrm{bA}^{\prime \prime}\right)$ halved \\
\hline $\mathrm{aA}^{\prime \prime}$ & 0.38 & 0.37 & 0.37 & 0.37 & 0.41 & 0.37 \\
\hline $\mathrm{bA}^{\prime}$ & 0.36 & 0.36 & 0.36 & 0.36 & 0.39 & 0.35 \\
\hline $\mathrm{cA}^{\prime}$ & 0.26 & 0.27 & 0.27 & 0.27 & 0.21 & 0.26 \\
\hline $\mathrm{bA}^{\prime \prime}$ & 0.00014 & 0.00025 & 0.00041 & 0.00063 & 0.00027 & 0.02 \\
\hline
\end{tabular}

a 1.5 ps lifetime to the three lowest states and to the ground state. The ps relaxation times observed by UV-vis time-resolved absorption can be attributed to the population equilibration of the lowest three states, their vibrational relaxation, and solvent restructuring $[14,15]$. SO-calculations shed some light also on the origin of the two excited-state IR signals, identity of the emissive state, and the temperature dependence of the emission lifetime, although no definitive explanation can be proposed yet. Two alternatives are possible:

(i) The major TRIR feature corresponds to the $\mathrm{aA}^{\prime \prime}$ and $\mathrm{bA}^{\prime}$ states, whereas the "minor" one is due to the third state $\mathrm{cA}^{\prime}$. The expected population ratio (Table 3) $73: 27$ is close to that observed experimentally [14] for $\left[\operatorname{Re}(\mathrm{Cl})(\mathrm{CO})_{3}\right.$ (phen)] in $\mathrm{MeCN}$, $80: 20$. Emission occurs from the third state $\mathrm{CA}^{\prime}$, while the

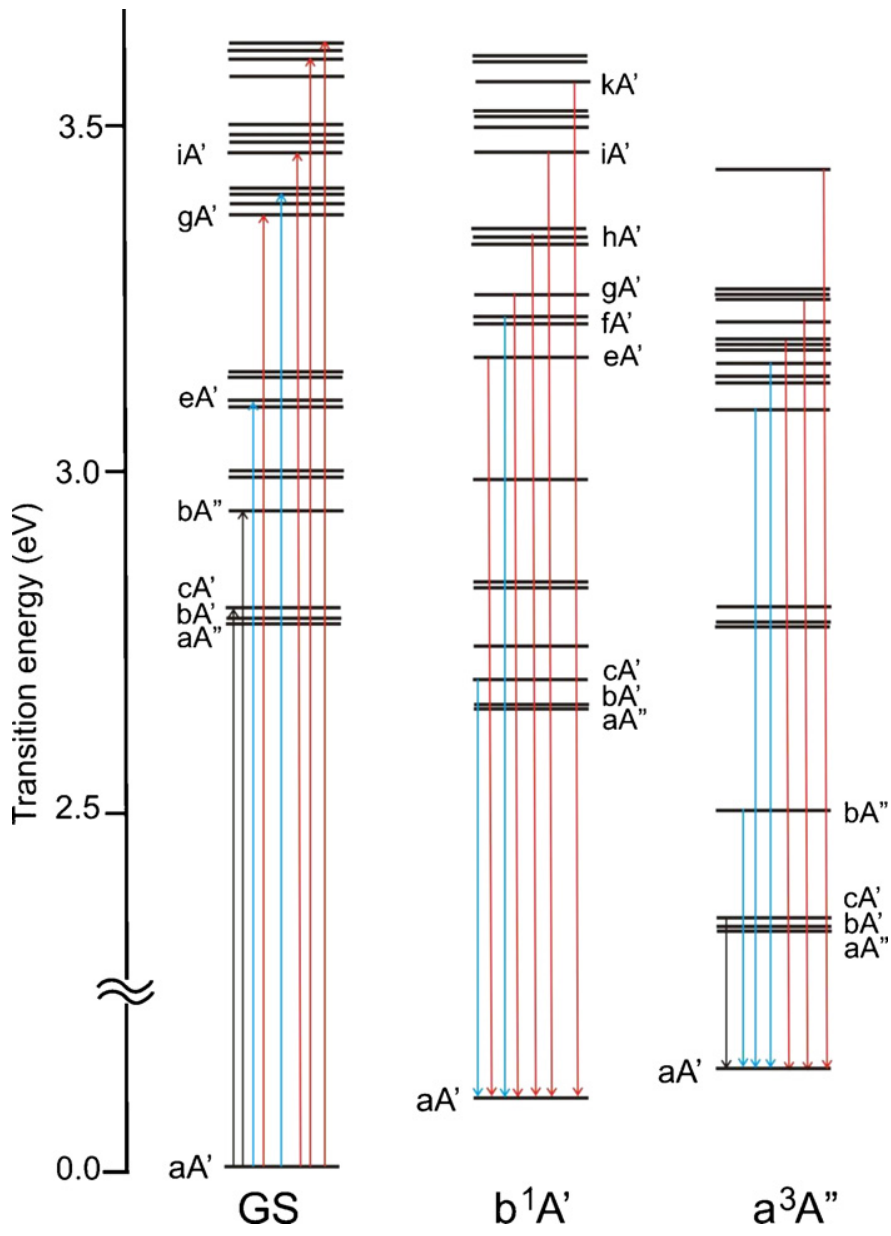

Fig. 6. SO states of $\left[\operatorname{Re}(\mathrm{imH})(\mathrm{CO})_{3}(\text { phen })\right]^{+}$in $\mathrm{DMF}$ solution calculated at the optimized geometries of the spin-free ground $a^{1} \mathrm{~A}^{\prime}$ (left), $\mathrm{b}^{1} \mathrm{~A}^{\prime}$ (middle) and $\mathrm{a}^{3} \mathrm{~A}^{\prime \prime}$ (right) states. Energies are referred to that of the $\mathrm{aA}^{\prime}$ ground state calculated at the $\mathrm{a}^{1} \mathrm{~A}^{\prime}$ geometry. Red, blue and black arrows indicate transitions with oscillator strengths larger than $0.01,0.001-0.01$ and $0.0005-0.001$, respectively. SO TDDFT (PBE0, COSMO-DMF) calculation. fourth state $\mathrm{bA}^{\prime \prime}$ provides the thermally activated decay pathway, ubiquitous in $\operatorname{Re}^{\mathrm{I}}$ carbonyl diimines $[81,82]$. However, this interpretation does not explain why the IR spectra of the "minor" and "major" states are so different [14].

(ii) The major excited-state IR signal belongs to the combined population of the three lowest MLCT states $a A^{\prime \prime}, b^{\prime}$, and $\mathrm{cA}^{\prime}$, whereas the "minor" one corresponds to the fourth state bA", lying at a lower energy than calculated. The long-lived emission occurs from the third $\left(\mathrm{cA}^{\prime}\right)$ and the fourth $\left(\mathrm{bA}^{\prime \prime}\right)$ state. (Note that $\mathrm{bA}^{\prime \prime}$ is the most emissive state amongst the low-lying SO states. Energy of relaxed $\mathrm{bA}^{\prime \prime}$ state is inevitably lower than that calculated herein at the $\mathrm{a}^{3} \mathrm{~A}^{\prime \prime}$ geometry.) The $\mathrm{bA}^{\prime \prime}$ state could also provide the thermally activated quenching pathway, if it undergoes a fast non-radiative decay to the ground-state. Alternatively, the thermal quenching could involve members of the next group of SO states, i.e. $\mathrm{cA}^{\prime \prime}, \mathrm{dA}^{\prime}$, or $\mathrm{dA}^{\prime \prime}$.

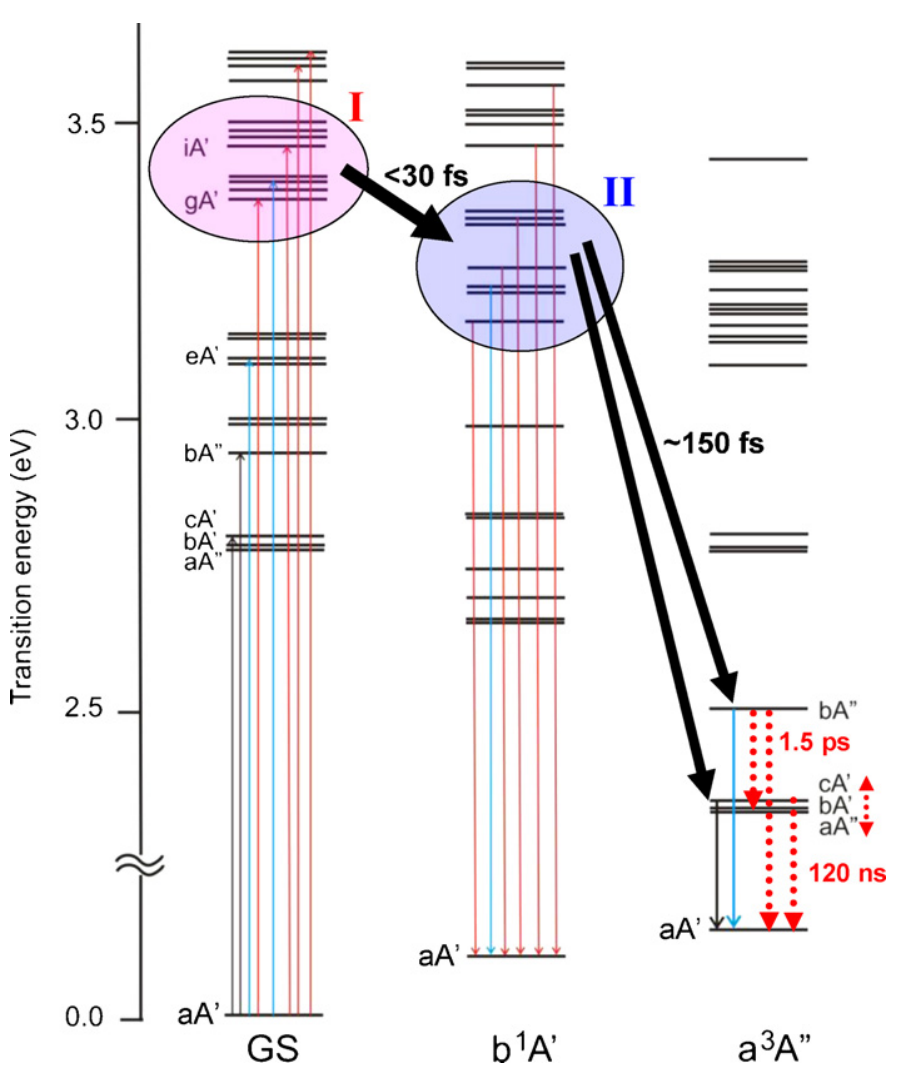

Fig. 7. Spin-orbit photophysical model of $\left[\operatorname{Re}(\mathrm{imH})(\mathrm{CO})_{3}(\text { phen })\right]^{+}$and other $\operatorname{Re}$ carbonyl-diimine complexes. Optical excitation populates the group of states I (red), followed by ultrafast electronic and vibrational energy redistribution to the group of states II, which undergoes prompt emission (red arrows) and $\sim 150$ fs conversion to bA" and the three lowest SO states, whose emission is shown by the thin black and blue arrows. Bold dotted red arrows denote nonradiative decay of the lowest SO states and the vertical double arrow stands for equilibration of the lowest three states. Vibrational relaxation is omitted from the diagram for the sake of clarity. (State energies are referred to that of the $\mathrm{aA}^{\prime}$ ground state calculated at the $\mathrm{a}^{1} \mathrm{~A}^{\prime}$ geometry. SO TDDFT (PBE0, COSMO-DMF) calculation.). 


\subsection{General aspects of the $\left[\operatorname{Re}(\mathrm{imH})(\mathrm{CO})_{3}(\text { phen })\right]^{+}$spin-orbit} model

The SO-model provides a comprehensive view of the $\left[\operatorname{Re}(\mathrm{imH})(\mathrm{CO})_{3}(\text { phen })\right]^{+}$excited-state behavior and accounts well for the principal experimental observations. Conclusions based on relativistic calculations are not only quantitatively, but also qualitatively different from the spin-free model, especially concerning the characters of the excited states involved and the identity of the intermediate state. The main conclusions emerging from this case study are generally valid for the large family of closed-shell heavy metal complexes:

(1) The SO excited-state density is much larger than assumed from the spin-free model. High excited-state density has several important consequences: (i) UV-vis absorption spectra originate from a large number of relatively weak transitions, (ii) emission can occur from several simultaneously populated SO states (e.g. $\mathrm{bA}^{\prime}, \mathrm{cA}^{\prime}$, and $\mathrm{bA}^{\prime \prime}$ in the present case), and (iii) crossings and conical intersections between closely spaced SO states could provide efficient electronic relaxation pathways via vibronic coupling.

(2) Majority of excited SO states cannot be classified as singlets or triplets because of their highly mixed-spin characters. The triplet character in the optically populated state increases upon structural relaxation of the excited molecule. The lowest three SO states largely retain the parent triplet character at both the ground-state and relaxed $\mathrm{a}^{3} \mathrm{~A}^{\prime \prime}$ geometries. The latter conclusion agrees with results of most (but not all) SO calculations (Section 4), as well as with experimental studies [1,2] of other heavy-metal complexes.

(3) Given the spin-mixed character of the emitting SO states, it is hardly possible to distinguish between fluorescence and phosphorescence and it is better to think in terms of prompt and long-lived emission or luminescence.

(4) In the presence of strong SOC, intersystem crossing and internal conversion should be treated in a unified way, as non-radiative transitions between SO states.

(5) Elucidating the mechanism of conversion between the SO states including dynamical effects such as vibronic coupling, and proposing selection rules is the next challenge. Solving this problem will allow us to specify the deactivation pathways and nonradiative transitions in terms of individual SO states, explain the specificity of photophysical and photochemical processes and achieve better control of excited-state dynamics and molecular photonic behavior.

\section{Overview of spin-orbit excited-state studies}

The SOC excited-state model of $\left[\operatorname{Re}(\mathrm{imH})(\mathrm{CO})_{3}(\text { phen })\right]^{+}$presented above is based either on calculations where SO is included in the Hamiltonian (RASSI-SO/MS-CASPT2) [71] or on a relativistic TDDFT approach (SO-TDDFT) [73]. These techniques calculate SO states and allow us to discuss absorption, fluorescence and phosphorescence, as well as intersystem crossing and internal conversion in a unified way. However, developing such excited-state schemes was made possible only by the most recent advances in computational quantum chemistry. It is not possible to infer such SO state diagrams from qualitative considerations and much simpler models are therefore often used to interpret experimental observations on excited-state properties and dynamics, Fig. 8. In parallel, advanced computational techniques are being developed to deal with SO effects at a high level of theory. In this section, we will briefly review these two research directions.

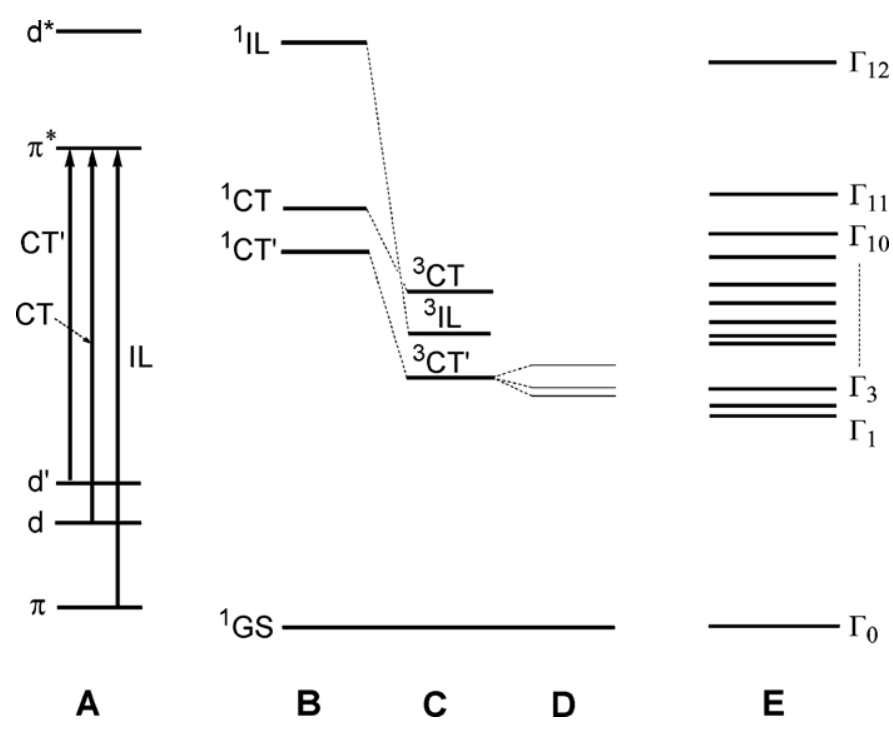

Fig. 8. Comparison of excited-state models of closed-shell heavy-metal complexes with electron accepting ligands. (A) One-electron excitations producing CT and IL states. (dd* excitation and LF states are not shown.) (B and C) Spin-free model consisting of distinct singlet (B) and triplet (C) excited-state manifolds. Note the typically much larger ${ }^{1} \mathrm{IL}-{ }^{3} \mathrm{IL}$ than ${ }^{1} \mathrm{CT}-{ }^{3} \mathrm{CT}$ separation. (D) Qualitative perturbational SO treatment predicts zero field splitting (ZFS) of the lowest triplet state to three sublevels. (E) Manifold of SO states resulting from relativistic calculations. Usually, the lowest three states $\Gamma_{1}-\Gamma_{3}$ can still be approximately viewed as the lowest-triplet sublevels, but higher SO states are strongly mixed and cannot be derived by splitting any particular triplet.

The discussion will be largely focused on closed-shell pseudooctahedral $\mathrm{d}^{6}$ or pseudo-square planar $\mathrm{d}^{8}$ complexes with electron-accepting $\mathrm{N}^{\wedge} \mathrm{N}$ or $\mathrm{C}^{\wedge} \mathrm{N}$ ligands, which are intensively studied because of their interesting photophysics and photonic applications [1-3]. As was already stated for the $\mathrm{Re}^{\mathrm{I}}$ complexes (Section 2), photophysics of this type of compounds are usually interpreted in terms of MLCT and IL states arising from $\mathrm{d} \rightarrow \pi^{*}$ and $\pi \rightarrow \pi^{*}$ one-electron excitations, respectively. More realistic excited-state description requires us to consider MLCT-LLCT mixing, if electron donating ligands are present in the coordination sphere alongside the $\mathrm{N}^{\wedge} \mathrm{N}$ or $\mathrm{C}^{\wedge} \mathrm{N}$ acceptors. Other types of excited states are of a little importance. LF states, due to $\mathrm{d} \rightarrow \mathrm{d}^{*}$ excitations, provide deactivation and reactive pathways in the case of $\mathrm{Ru}^{\mathrm{II}}$ polypyridines [85-87], while, in 3rd-row transition metal complexes, they occur at too high energies to play any important photophysical role. Moreover, they are highly mixed with other excitations and the $\mathrm{d}$ and $\mathrm{d}^{*}$ orbitals themselves are rather delocalized $[9,88,89]$. (Note, however, that LF states are of a paramount importance in photophysics of 3d-metal complexes, e.g. $\left[\mathrm{Fe}(\mathrm{bpy})_{3}\right]^{2+}[90,91]$ or $\mathrm{Cr}(\text { acetylacetonate })_{3}$ [92].)

\subsection{Qualitative approach to spin-orbit coupling}

Instead of developing a full scheme of SOC states (such as that shown in Figs. 2, 3-center and, schematically, 8E), qualitative models start with separate manifolds of spin-free singlet and triplet states and apply SOC as a small perturbation that mixes the lowest triplet state with singlet states as well as with other triplets $[1-3,10,93,94]$. As a result, the lowest triplet-state $T_{1}$ splits to three spin sublevels $T_{1(i)}$ whose energies $E_{i}$ are given by Eq. (4):

$E_{i}=E_{T_{1}}+\sum_{S_{n}} \frac{\left|\left\langle S_{n}\left|\hat{H}_{s o}\right| T_{1(i)}\right\rangle\right|^{2}}{E_{T_{1}}-E_{S_{n}}}+\sum_{T_{n}} \frac{\left|\left\langle T_{n(j)}\left|\hat{H}_{s o}\right| T_{1(i)}\right\rangle\right|^{2}}{E_{T_{1}}-E_{T_{n}}}$

$E_{T_{1}}$ is the energy of the unperturbed spin-free lowest triplet state, $S_{n}$ are the wavefunctions of spin-free singlet states, $T_{n}$ are higher 
triplet states, and $T_{n(j)}$ are wavefunctions of their SO sublevels. The $S_{n}$ state and a the triplet sublevel $T_{1(i)}$, as well as the sublevels $T_{n(j)}$ and $T_{1(i)}$ must have the same double-group symmetry for $\left\langle S_{n}\left|\hat{H}_{s o}\right| T_{k(i)}\right\rangle$ and $\left\langle T_{n(j)}\left|\hat{H}_{s o}\right| T_{1(i)}\right\rangle$ to be nonzero, respectively.

Eq. (4) shows that ZFS of the $T_{1}$ state results from SO-induced mixing of the spin-free triplet $T_{1}$ with singlets $S_{n}$ (the first term), and with substates of other triplets (the second term). The singlet-triplet SO mixing determines the radiative rate constant for emission of the sublevel $T_{1 i}$ :

$k_{r}(i) \cong$ const $\times \tilde{v}^{3} \times\left|\sum_{S_{n}} \frac{\left\langle S_{n}\left|\hat{H}_{\mathrm{SO}}\right| T_{1(i)}\right\rangle}{E_{T_{1}}-E_{S_{n}}} \times\left\langle S_{0}|\overrightarrow{e r}| S_{n}\right\rangle\right|^{2} \times \mathrm{FC}$

It follows that the radiative decay rate depends not only on the singlet-triplet SO mixing, but also on the transition moments $\left\langle S_{0}|\vec{r}| S_{n}\right\rangle$ of the admixed singlet transitions, and on the vibrational overlap (i.e. the Franck-Condon factor, $F C$ ). This is nicely illustrated by the $\mathrm{eA}^{\prime}$ state of $\left[\operatorname{Re}(\mathrm{imH})(\mathrm{CO})_{3}(\text { phen })\right]^{+}$(Table 1$)$ that is predominantly triplet but partially spectroscopically allowed (osc. strength $=0.0017$ ) due to only $2 \%$ admixture of the strongly allowed $\mathrm{b}^{1} \mathrm{~A}^{\prime}$ spin-free state. On the other hand, the transition to $\mathrm{dA}^{\prime \prime}$ has oscillator strength of only 0.0004 despite having $57 \%$ singlet contribution, but from a spectroscopicly strongly forbidden spin-free state $\mathrm{a}^{1} \mathrm{~A}^{\prime \prime}$.

Eq. (4) can be, in principle, applied to any triplet state $T_{k}$ and its sublevels $T_{k(i)}$. Estimating SOC matrix elements $\left\langle S_{n}\left|\hat{H}_{s o}\right| T_{k}\right\rangle$ allows us to single out strongly SO-coupled states and thus identify fast ISC pathways or strongly emissive states. Such estimates are based on the following approximations: (i) Only the one-electron $\hat{H}_{\text {So }}$ term (Eq. (2)) is considered. (ii) Only contributions of the metal atom and heavier halogen ligands, if present in the coordination sphere, are considered. Contributions from all light atoms, such as $\mathrm{H}, \mathrm{C}, \mathrm{O}$, $\mathrm{N}$, etc. are neglected because of the pronounced $\xi\left(r_{i A}\right)$ dependence on the nuclear charge, Eq. (3). (iii) Excited states are expressed as linear combinations of one-electron excitations between KS molecular orbitals (Eq. (1a)). (iv) Only one-center SO interactions are considered, i.e. SOC induced by the nuclear charge $\left(Z_{A}^{\text {eff }}\right.$, Eq. (3)) of atom $A$ acts only on the electron density localized at the atom $A$, described the corresponding atomic orbital wavefunction times the LCAO mixing coefficient in the KS molecular orbitals that are involved in the excitation.

Using these approximations and an empirical knowledge of the behavior of IL and MLCT excited states, it is possible to formulate qualitative rules to identify strongly SO-coupled states $[1-3,16,17,93,94]$ :

1. Separation between spin-free ${ }^{1} \mathrm{IL}$ and ${ }^{3} \mathrm{IL}$ states is much larger than between ${ }^{1}$ MLCT and ${ }^{3}$ MLCT. Consequently, ${ }^{3}$ IL states often occur energetically below ${ }^{1} \mathrm{MLCT}$ and close to ${ }^{3} \mathrm{MLCT}$, even if ${ }^{1} \mathrm{IL}$ lies at high energies. (This is an effect of the exchange integral $K$, not SOC.)

2. ${ }^{1,3}$ MLCT energies are much more environment-dependent than

1,3 IL ones. Increasing the solvent polarity or going from solvents to rigid matrices increase the ${ }^{3}$ MLCT energy, pushing ${ }^{3} \mathrm{MLCT}$ states closer to, or even above ${ }^{3}$ IL. TDDFT in vacuo calculates much lower ${ }^{1,3}$ MLCT energies than in the case that the solvent is included through dielectric models $[9,61]$.

3. SOC between any two spin-free singlet and triplet states $S_{n}$ and $T_{k}$ is determined by the SO matrix element $\left\langle S_{n}\left|\hat{H}_{\mathrm{SO}}\right| T_{k}\right\rangle$ that will have a large value if $S_{n}$ and $T_{k}$ differ in one spinorbital, which in each state has to be largely localized on the metal and/or other heavy atom(s). Hence, strong ${ }^{1} \operatorname{MLCT}\left(\mathrm{d} \pi^{*}\right) \leftrightarrow{ }^{3} \operatorname{MLCT}\left(\mathrm{d}^{\prime} \pi^{*}\right)$ SOC is expected, because the two states differ only in the depopulated orbital ( $\mathrm{d}$ and $\mathrm{d}^{\prime}$, see Fig. $8 \mathrm{~A}$ ) that is in each case metal-localized. On the other hand, ${ }^{1} \mathrm{IL} \leftrightarrow{ }^{3} \mathrm{IL}$ SOC will be weak because the two states arise from the same $\pi \pi^{*}$ excitation and both orbitals are largely localized on light ligand atoms. ${ }^{1} \operatorname{IL}\left(\pi \pi^{*}\right) \leftrightarrow{ }^{3} \operatorname{MLCT}\left(\mathrm{d} \pi^{*}\right)$ and ${ }^{1} \operatorname{MLCT}\left(\mathrm{d} \pi^{*}\right) \leftrightarrow{ }^{3} \operatorname{IL}\left(\pi \pi^{*}\right)$ interactions also will be weak even if they differ in one depopulated spin orbital, $\mathrm{d} v s$. $\pi$. This is because the $\mathrm{d}$ and $\pi$ orbitals are localized at different parts of the molecule, in contrary to the assumption (iv) above. (Nevertheless, ${ }^{3}$ IL states are subjected to SOC effects through their interconfigurational mixing with ${ }^{3}$ MLCT, see Section 2.)

4. An SO matrix element $\left\langle S_{n}\left|\hat{H}_{\mathrm{SO}}\right| T_{k}\right\rangle$ between spin-free states will have a non-zero value only if the direct product of $S_{n}$ and $T_{k}$ symmetry representations transforms as one of the rotation components $R_{x}, R_{y}$, or $R_{z}$. Therefore, a ${ }^{1}$ MLCT $\leftrightarrow{ }^{3}$ MLCT SOC will be strong only if the two states differ in the depopulated $\mathrm{d}$ orbital, the electron-accepting $\pi^{*}$ orbital being the same. In terms of Fig. 8, this means a strong SOC between the ${ }^{1} \operatorname{MLCT}\left(\mathrm{d} \pi^{*}\right)$ and ${ }^{3} \operatorname{MLCT}\left(\mathrm{d}^{\prime} \pi^{*}\right)$ states, but not between ${ }^{1} \operatorname{MLCT}\left(\mathrm{d} \pi^{*}\right)$ and ${ }^{3} \operatorname{MLCT}\left(\mathrm{d} \pi^{*}\right)$. Similar argument can be made for SOC between halide-to-diimine ${ }^{1,3}$ LLCT states that have to involve different halide $\mathrm{p}_{\pi}$ orbitals in the triplet and the singlet [10].

5. Having in hand DFT-calculated KS molecular orbitals and TDDFT-calculated spin-free states, it is possible to estimate the magnitude of a SOC matrix element between states $S_{n}$ and $T_{k}$ whose wavefunctions contain contributions from $\mathrm{MO} \rightarrow \pi^{*}$ and $\mathrm{MO}^{\prime} \rightarrow \pi^{*}$ MLCT excitations, respectively:

$\left\langle S_{n}\left|\hat{H}_{\mathrm{SO}}\right| T_{k}\right\rangle=a b \xi_{M}\left(c_{\mathrm{d}} c_{\mathrm{d}}^{\prime}\right)\left\{\left\langle\mathrm{d}|\hat{l} \cdot \hat{s}| \mathrm{d}^{\prime}\right\rangle\right\}$

$c_{\mathrm{d}}$ and $c_{\mathrm{d}}^{\prime}$ are the $\mathrm{d}$ and $\mathrm{d}^{\prime}$ LCAO coefficients in the occupied molecular orbitals MO and MO', respectively; $a$ and $b$ stand for the weights of $\mathrm{MO} \rightarrow \pi^{*}$ and $\mathrm{MO}^{\prime} \rightarrow \pi^{*}$ MLCT excitations in the $S_{n}$ and $T_{k}$ states, respectively, in the spirit of Eq. (1). The last term depends on the molecular symmetry and contains SO integrals between $\mathrm{d}$ and $\mathrm{d}^{\prime}$ spinorbitals whose values are tabulated [95]. Eq. (6) predicts SO between given spin-free singlet and triplet states to increase with increasing d-orbital participation in the molecular orbitals involved $\left(c_{\mathrm{d}}\right.$ and $\left.c_{\mathrm{d}}^{\prime}\right)$ and with increasing MLCT excited-state characters $a$ and $b$. This equation thus takes care of both the orbital delocalization and excitation mixing.

Rules 1-5 have been expanded and used to identify strongly emitting metal complexes applicable in OLEDs and formulate useful design rules. This subject is very well covered in the literature $[1-3,16,17]$.

The use of these qualitative assumptions and rules can be illustrated, for example, by a search for strongly SO coupled states and ISC pathways in $\mathrm{Re}^{\mathrm{I}}, \mathrm{Cu}^{\mathrm{I}}$, and $\mathrm{Pt}^{0}$ complexes:

The SO matrix element $\left\langle b^{1} A^{\prime}\left|\hat{H}_{\text {SO }}\right| a^{3} A^{\prime \prime}\right\rangle$ of Re carbonyl-diimines was estimated [10] as $a b(-i / 2) \xi_{\text {Re }}\left(c_{d}^{\prime} c_{d}^{\prime \prime}\right)$ and $a b(-i / 2)\left(\xi_{\mathrm{Re}} c_{\mathrm{d}}^{\prime} c_{\mathrm{d}}^{\prime \prime}+\right.$ $\left.\xi_{X} c_{\mathrm{p}}^{\prime} c_{\mathrm{p}}^{\prime \prime}\right)$ for $\left[\operatorname{Re}(\mathrm{Etpy})(\mathrm{CO})_{3}(\mathrm{bpy})\right]^{+}$and $\left[\operatorname{Re}(\mathrm{X})(\mathrm{CO})_{3}(\mathrm{bpy})\right](\mathrm{X}=\mathrm{Cl}, \mathrm{I})$, respectively. $c_{d}^{\prime}$ and $c_{p}^{\prime}$ are the $d(R e)$ and $p(X)$ LCAO coefficients in the HOMO- 1 , while $c_{\mathrm{d}}^{\prime \prime}$ and $c_{\mathrm{p}}^{\prime \prime}$ are the corresponding coefficients in the HOMO, $\xi$ is the atomic spin-orbit parameter [96], $a$ and $b$ are the weights of the HOMO- $\rightarrow$ LUMO and HOMO $\rightarrow$ LUMO excitations in the transitions to the $b^{1} \mathrm{~A}^{\prime}$ and $\mathrm{a}^{3} \mathrm{~A}^{\prime \prime}$ states in the sense of Eq. (1), respectively. The square SO matrix element values of 550 , 503 and $1303 \mathrm{~cm}^{-1}$ obtained for the Etpy, $\mathrm{Cl}$, and I species, respectively, demonstrate the importance of heavy halogen atoms, i.e. of the $\mathrm{X} \rightarrow$ bpy LLCT contribution, for SOC [10]. Smaller values (58, 92 , and $267 \mathrm{~cm}^{-1}$, respectively) were obtained for $\left\langle b^{1} A^{\prime}\left|\hat{H}_{\mathrm{SO}}\right| b^{3} A^{\prime \prime}\right\rangle$, because of much smaller MLCT weight $b$ in the $\mathrm{b}^{3} \mathrm{~A}^{\prime \prime}$ spin-free state.

A similar analysis of tetracoordinate $\mathrm{Cu}^{\mathrm{I}}$ and $\mathrm{Pt}^{0}$ complexes, whose optically populated ${ }^{1}$ MLCT state undergoes surprisingly slow ISC (13-16 and 3.2 ps, respectively), revealed that the SO matrix elements between the ${ }^{1}$ MLCT and low-lying triplets are very small because they involve the same d orbital $[93,94]$. Strongly coupled triplets lie at inaccessibly high energies and cannot engage in ISC. Vice versa, the lowest triplets are well SO-coupled only to 
high-lying singlet states that are not populated at the excitation wavelengths used. ISC rates are limited by the small SOC between the optically excited ${ }^{1}$ MLCT and the lowest triplet states. Energetic inaccessibility of suitable SO-interacting singlets also explains the slow ISC in the dinuclear complex $\left[\mathrm{Pt}_{2}(\mathrm{pop})_{4}\right]^{4-}[97-99]$.

The ZFS concept (Eq. (4)) and qualitative SOC treatments outlined in this section provide a convenient way to discuss and understand the behavior of the lowest triplet state and predict emission from its sublevels using Eq. (5). Higher states engage in so many strong SO interactions that they should be regarded as SO states instead of sublevels of any parent spin-free triplet. Still, their energies and compositions in terms of parent spin-free states can be obtained perturbationally, but the full SO secular problem has to be solved [100], see Section 4.2. This procedure is necessary even for the lowest triplet and its sublevels, if the ZFS is to be calculated accurately. For example, the $\mathrm{T}_{1}$ state of $\operatorname{Ir}(\mathrm{ppy})_{3}$ interacts strongly with at lest 8 singlet states [101]. 50 spin-free singlets and 50 triplets had to be included in perturbational TDDFT calculations of $\operatorname{Ir}(\mathrm{ppy})_{3},\left[\mathrm{Ru}(\mathrm{bpy})_{3}\right]^{2+}$, and $\left[\mathrm{Os}(\mathrm{bpy})_{3}\right]^{2+}$ to achieve a good agreement with experimental ZFS [100].

\subsection{Spin-orbit excited-state calculations}

The first theoretical studies of SOC effects on MLCT excited states emerged in early 1980s. SOC models of $\left[\mathrm{M}(\mathrm{bpy})_{3}\right]^{2+}(\mathrm{M}=\mathrm{Fe}, \mathrm{Ru}, \mathrm{Os})$ electronic structures and spectra [102-104] have formulated the SO splitting patterns, SOC matrix elements, and spin-state symmetries and wavefunctions. Similar approach was applied $[105,106]$ to phosphorescent ${ }^{3} \mathrm{IL}$ states of $\left[\mathrm{Rh}(\mathrm{bpy})_{3}\right]^{3+}$ and $\left[\mathrm{Rh}(\mathrm{phen})_{3}\right]^{3+}$ with a much smaller ZFS, and to the metal-metal bonded complex $\left[\mathrm{Pt}_{2}\left(\mathrm{P}_{2} \mathrm{O}_{5} \mathrm{H}_{2}\right)_{4}\right]^{2-}[98]$. A DFT computational study of $\left[\mathrm{Ru}(\mathrm{bpy})_{3}\right]^{2+}$ appeared in 1994, which included SOC into DFT from the first principles (i.e. not as an additional perturbation), the Ru spin-orbit coupling constant having been the only empirical parameter used [107]. This work has provided the first quantitative SO model of $\left[\mathrm{Ru}(\mathrm{bpy})_{3}\right]^{2+}$ excited states and its results compared reasonably well to the experimental absorption spectrum.

In-depth SO computational studies of metal complexes are still rather rare. Spectroscopic SO effects were investigated for $\mathrm{H}_{2} \mathrm{M}(\mathrm{CO})_{4}(\mathrm{M}=\mathrm{Fe}$, Os $)$ [18]. CASSCF/MS-CASPT2 calculations yielded a manifold of $\mathrm{M} \rightarrow \mathrm{CO}$ MLCT SO states, revealed a decrease of absorption-band intensities and, for $\mathrm{H}_{2} \mathrm{Fe}(\mathrm{CO})_{4}$, an emergence of a new spectroscopic feature upon including SOC. Some of the $\mathrm{H}_{2} \mathrm{Os}(\mathrm{CO})_{4}$ spin-free triplets are split and mixed with other states to such an extent that they cannot be related to any particular set of spin-free triplet states. For example, the spin-free $a^{3} B_{2}$ state is split by $\sim 3000 \mathrm{~cm}^{-1}$, contributing $40-50 \%$ to several SO states. Interesting are CASSCF-type calculations of SOC effects on nonradiative photophysical and photochemical behavior, for example, intersystem crossing in $\mathrm{HCo}(\mathrm{CO})_{4}$ [108], $\mathrm{M}-\mathrm{H}$ bond photodissociation in $\left[\mathrm{M}(\mathrm{H})(\mathrm{CO})_{3}\right.$ (1,4-diazabutadiene)] [109], and intraligand trans-cis isomerization in $\left[\operatorname{Re}(\text { styrylpyridine })(\mathrm{CO})_{3}(\mathrm{bpy})\right]^{+}[19,110]$. Emission from SO states has been investigated for OLED-relevant $\mathrm{Pd}(\text { thpy })_{2}$ and $\mathrm{Pt}(\text { thpy })_{2}$ cyclometallated complexes by relativistic multiconfiguration SCF [111]. Much larger singlet-triplet mixing and, hence, emission transition moments were calculated for $\mathrm{Pt}$ than Pd, not only because of larger Pt effective nuclear charge $Z_{\text {eff }}$ but also due to a higher $\mathrm{d}(\mathrm{Pt})$ orbital participation in the MOs depopulated upon excitation (i.e. due to a higher MLCT content in $\mathrm{Pt}(\text { thpy })_{2}$ excited states). The calculation has produced a series of Pt(thpy $)_{2}$ SO states together with emission transition moments. The first two SO states can be regarded as sublevels of the lowest triplet while the third SO state is calculated highly mixed and $\sim 700 \mathrm{~cm}^{-1}$ above the second one. All SO excited states except the lowest one are predicted to be emissive, but most of the emission will originate from the second SO state, since the higher ones lie at too high energies to be thermally populated after equilibration. In addition, potential-energy curves of SO states along a ligand twisting coordinate were calculated, together with energies and intensities of emission from individual vibronic SO states. This work provides an interesting insight into the properties of SO states, although the results on the third state do not agree with experiments [1] that found the third SO state only $9 \mathrm{~cm}^{-1}$ above the second one and showed that all three lowest-lying SO states behave as the lowest-triplet sublevels.

A perturbational TDDFT approach to SOC has been used to calculate SOC-states of $\operatorname{Ir}(\mathrm{ppy})_{3}[100,101], \operatorname{Ir}(\mathrm{ppy})_{2} \mathrm{~L}$ [112], cyclometallated $\mathrm{Pt}^{\mathrm{II}}$ complexes [16], as well as $\left[\mathrm{M}(\mathrm{bpy})_{3}\right]^{2+}(\mathrm{M}=\mathrm{Ru}$, Os) [100]. TD-DFT was first used to calculate the energies and wavefunctions of spin-free singlet and triplet states. SO state energies and compositions were then obtained by constructing the SO matrix, that describes all possible SO interactions between the spin-free states, and solving the SO secular equation [100]. These calculations revealed very high densities of SO states, identified principal coupling pathways, estimated ZFS, and provided absorption and emission transition energies and oscillator strengths. It has been noted [100] that both optical excitation and electron-hole recombination in $\operatorname{Ir}(\mathrm{ppy})_{3}$ populate directly SO states, which are therefore relevant to OLED operation.

\section{Experimental observations of spin-orbit phenomena}

Zero-field splitting (ZFS), strong "phosphorescence" (i.e. fast triplet radiative decay), ultrafast triplet-state population (ISC), and fast nonradiative decay from the lowest triplet to the ground state are the most obvious photophysical manifestations of SOC. Ultrafast excited-state dynamics are expected to be affected as well, the high density of SO states providing efficient electronic relaxation pathways.

To interpret experimental excited-state behavior, we can use the spin-free model, the qualitative perturbational SO approach or, more rigorously, we can work within the framework of SO states, as explained in Section 3. Spin-free calculations are widely used to assign UV-vis absorption spectra of heavy metal complexes, often achieving good quantitative match with experimental spectra. However, Fig. 4 clearly demonstrates that spin-free and SO assignments of the $\left[\operatorname{Re}(\mathrm{imH})(\mathrm{CO})_{3}(\text { phen })\right]^{+}$lowest absorption band are qualitatively different, despite close resemblance of the two simulated spectra. SOC explains better the broad shapes of absorption bands of heavy-metal complexes, attributing them to a multitude of transitions to a larger number of SO states. Similar statement can be made about broad emission bands, although the number of emitting SO states is limited by Botzman population distribution. Assigning absorption and emission spectra according to the SO-model could have important implications for evaluating molecular excited-state distortion from spectroscopic band shapes (e.g. the Huang-Rhys factor) and interpreting resonance Raman spectra.

The spin-free model works with unrealistically low excitedstate densities. In the $\left[\operatorname{Re}(\mathrm{imH})(\mathrm{CO})_{3}(\text { phen })\right]^{+}$example, there are 5 spin-free states ( 2 singlets, 3 triplets) between the lowest triplet $a^{3} \mathrm{~A}^{\prime \prime}$ and lowest allowed singlet $\mathrm{b}^{1} \mathrm{~A}^{\prime}$, in contrast with 14 SO states calculated in the same energy range, Fig. 3. $\left[\operatorname{Re}(\mathrm{imH})(\mathrm{CO})_{3}(\text { phen })\right]^{+}$ is a relatively simple chromophore with only single electronaccepting ligand. The situation becomes much more complicated in complexes like Ir(ppy $)_{3}$, where 70 SO states were calculated within $1 \mathrm{eV}$ above the lowest excited state (or 140 states within $1.88 \mathrm{eV}$ ) [100]. The presence of so many excited states in a narrow energy range must have dramatic consequences for absorption and emission spectra, as well as electronic relaxation following excitation. 
Spin-free model alone is incapable of interpreting ZFS or phosphorescence but it can be easily amended by including SOC as a weak perturbation, Eqs. (4) and (5). In the full SO-model, ZFS is just a phenomenological parameter, defined as the energy difference between the lowest three SO states. Instead of fluorescence and phosphorescence, we talk about emission (photoluminescence) from individual SO states. The radiative rate constant of emission from an excited state $\mathrm{SO}_{m}$ to the ground state $\mathrm{SO}_{0}$ is expressed [111] by Eq. (7),

$k_{r}(m) \cong$ const $\times \tilde{v}^{3} \times\left\langle S_{m}|e \hat{r}| S O_{0}\right\rangle^{2} \times \mathrm{FC}$

which describes the transition probability using the usual transition moment. SO matrix elements do not enter the equation since $\mathrm{SOC}$ is already included in the wavefunctions $\mathrm{SO}_{m}$ and $\mathrm{SO}_{0}$. Similarly, intersystem crossing and internal conversion are no longer distinguished and are treated in a unified way as nonradiative transitions between SO states. However, a satisfactory theory is still missing.

In the following text, we will briefly comment on the main photophysical manifestations of SOC.

Zero-field splitting. The ZFS concept is well applicable to the lowest three SO states that can be regarded as sublevels of the lowest spin-free triplet state. ZFS is an experimental parameter that can be obtained from high-resolution emission spectra at low temperatures or temperature-dependent emission lifetimes [1,2]. Very small ZFS in Rh complexes was measured by optically detected magnetic resonance $[105,106]$. Experimental ZFS shows the energy separation of the three lowest SO states and is most often interpreted using the perturbational language (Eq. (4)) as the splitting of the lowest triplet state. Substantial SOC-induced ZFS of tens of $\mathrm{cm}^{-1}$ is very common in transition metal polypyridine and cyclometallated complexes [1,2]. ZFS magnitude increases with increasing d-orbital participation in the emitting triplet, i.e. with its MLCT character. Large ZFS of a metal complex indicates strong phosphorescence and potential applicability in OLEDs [1,2].

Site-selective emission spectroscopy in solid matrices has revealed that ZFS can vary by as much as $100 \%$ between different sites of the matrix $[1,2,113]$. This observation shows that the MLCT character of the lowest triplet, which is mostly determined by the ${ }^{3} \mathrm{MLCT}^{3}{ }^{3} \mathrm{IL}$ mixing, is very sensitive to the molecular environment. It is not known experimentally how ZFS changes on going from a solid matrix to a fluid solution and how it depends on the solvent. Nevertheless, the well-known "rigidochromic effect" [114] demonstrates that ${ }^{3}$ MLCT states lie at lower energies in solutions than in glasses, further apart from the ${ }^{3}$ IL states. Larger ${ }^{3}$ MLCT $-{ }^{3}$ IL energy separation in solution results in smaller mixing and, hence, larger MLCT content in the lowest triplet. This conclusion is supported by the observation of an "IR rigidochromism", when excited-state $v$ (CO) IR bands of Re carbonyl-diimines occur at higher energies in solutions than low-T glasses [66]. Therefore, we may expect ZFS of emissive ${ }^{3}$ MLCT states to be larger in fluid solutions than the values reported from low-temperature experiments. It can even be expected that the ${ }^{3} \mathrm{IL}-{ }^{3}$ MLCT mixing and, hence, ZFS changes during relaxation of the excited chromophore and its environment, as was suggested for Re ${ }^{I}$ carbonyl-diimines by TRIR [15,51,63-65].

ZFS strongly affects low-temperature emission spectra and decay, because of an unequal and temperature-dependent sublevel population. Out of many known cases, some representative examples are discussed in refs. [1,2,83,84,113,115-117]. Sublevels derived from the lowest triplet have very different emission lifetimes due to different radiative and nonradiative decay rates. The lowest sublevel usually has the highest triplet content and is the longest-lived one, whereas the highest sublevel (i.e. the third SO state) is most emissive $[1,2,115,116]$. Equilibration between sublevels in low-temperature matrices is in most cases faster than the decay times, i.e. sub-microsecond. Such complexes then exhibit thermally equilibrated emission and pronounced temperature dependences of emission lifetimes. At very low temperatures $(<2 \mathrm{~K})$, only the lowest sublevel (i.e. the first SO state) is populated and its lifetime can be determined separately [1,2,116,117]. Interconversion between the sublevels in low-T matrices is treated as spin-lattice relaxation and its mechanisms have been thoroughly reviewed [1]. Only few kinetics data are available. For example, in $\left[\mathrm{Pt}(\mathrm{thpy})_{2}\right]$, the conversion of the second to the first sublevel over a $7 \mathrm{~cm}^{-1}$ energy difference takes place with a $720 \mathrm{~ns}$ lifetime at $1.3 \mathrm{~K}$, whereas conversions of the third sublevel to the second and first ones are much faster [1]. Very slow conversion of the second to the first triplet sublevel, $22 \mu \mathrm{s}$, was measured for [ $\operatorname{Ir}\left(\right.$ bis $\left(2-\left(2^{\prime}\right.\right.$-benzothienyl)-pyridinato- $\left.\mathrm{N}, \mathrm{C}^{3 \prime}\right)$ (acetylacetonate)] at $1.5 \mathrm{~K}[1,113]$. A Pt ${ }^{\mathrm{II}}$ complex $\left[\mathrm{Pt}(4,6\right.$-difluoro-ppy $\left.)(\mathrm{CN})_{2}\right]$ is an exceptional case [115] exhibiting a triexponential emission decay $(11.5,31.5$, and $100 \mu \mathrm{s})$ at $1.2 \mathrm{~K}$ from non-thermalized lowest triplet sublevels that are separated by less than $2 \mathrm{~cm}^{-1}$, whereas a single exponential decay $(28.3 \mu \mathrm{s})$ was measured at $10 \mathrm{~K}$. It follows that the spin-lattice relaxation is very slow at $1.2 \mathrm{~K}$ but gets much faster as the temperature increases. Interconversion between the lowest-triplet sublevels (i.e. the lowest three SO states) will be much faster in fluid solutions at ambient temperature because of high density and accessibility of energy-accepting vibrational states and solvent fluctuations that help overcoming energy barriers. For example, equilibration of two simultaneously populated SO states of $\left[\operatorname{Re}(\mathrm{Cl})(\mathrm{CO})_{3}\left(\mathrm{~N}^{\wedge} \mathrm{N}\right)\right]$ has been found to take only a few ps in MeCN [14].

ZFS effects on ambient-temperature photophysics are usually neglected, which could lead to erroneous predictions and obscure the real origin of the observed photophysical phenomena. This point can be illustrated using $\left[\operatorname{Re}(\mathrm{Cl})(\mathrm{CO})_{3}\right.$ (phen)] as an example. Its lowest ${ }^{3} \mathrm{CT}$ state is split into three sublevels [83,84]. The two higher ones $\mathrm{T}_{\text {III }}$ and $\mathrm{T}_{\text {II }}$ lie 42.1 and $14 \mathrm{~cm}^{-1}$ above $\mathrm{T}_{\mathrm{I}}$, respectively (EPA glass, $T<50 \mathrm{~K}$ ). The substate lifetimes differ by 2 orders of magnitude: $149,34.5$, and $1.92 \mu \mathrm{s}$, respectively. The three distinct decay processes are manifested by the strong temperature dependence of the emission decay lifetime below $50 \mathrm{~K}$. Such low-T photophysical phenomena are normally assumed to be leveled off as the temperature increases, since the thermal energy $k_{\mathrm{B}} T$ is larger than ZFS and sublevel equilibration occurs on a fs-ps timescale. However, ZFS in fluid solutions could be larger than in low-T matrices and give rise to T-dependent emission even at ambient temperatures. More importantly, there is no reason to assume that emission originates only from the lowest-triplet sublevels. For example, in the case of $\left[\operatorname{Re}(\mathrm{imH})(\mathrm{CO})_{3}(\mathrm{phen})\right]^{+}$, we have found three close-lying emissive states $\mathrm{bA}^{\prime}, \mathrm{cA}^{\prime}$ and bA", Figs. 6 and 7. The latter is the 4th SO state, not a lowest-triplet sublevel. Several emissive states could be populated in more complex OLED-relevant chromophores, although direct experimental evidence is lacking and theoretical calculations would require structural optimization of individual SO states - a very difficult task, indeed. Simultaneous emission from several SO states has to be considered when designing spectroscopically pure OLED emitters.

Emission polarization. Anisotropy of emission from an SO state could be different from that predicted for phosphorescence from the lowest spin-free triplet state, because the most emissive sublevel could have a different symmetry. For example, the $\left[\operatorname{Re}(\mathrm{imH})(\mathrm{CO})_{3}(\text { phen })\right]^{+}$lowest spin-free triplet is $\mathrm{a}^{3} \mathrm{~A}^{\prime \prime}$ (Figs. 2 and 3-right), predicting a perpendicular phosphorescence polarization that would be manifested by anisotropy of -0.2 . Experimentally, values slightly lower than +0.4 were found for $\left[\operatorname{Re}(\mathrm{imH})(\mathrm{CO})_{3}(\text { phen })\right]^{+}(0.4)$ and $\left[\operatorname{Re}(\mathrm{imH})(\mathrm{CO})_{3}(\mathrm{dmp})\right]^{+}(0.2-0.3)$ bound to proteins $[51,118]$ and for $\left[\operatorname{Re}(\mathrm{Et}-\mathrm{imH})(\mathrm{CO})_{3}(\text { phen })\right]^{+}$in a MeCN solution (ca. 0.4). Emission anisotropy of 0.3 was measured [119] for a similar complex $\left[\operatorname{Re}(4-\mathrm{HOOC}\right.$-pyridine $)(\mathrm{CO})_{3}\left(2,9-\mathrm{Me}_{2}-\right.$ 
4,7- $\mathrm{Ph}_{2}$-phen) $]^{+}$in a glycerol glass. These anisotropy values indicate that the absorption and emission transition moments are nearly parallel (theoretical anisotropy of 0.4 ). Since light absorption excites transitions to $\mathrm{A}^{\prime}$ SO states (Fig. 3, Table 1 ), the observation of a nearly parallel polarization shows that emission occurs from an SO state of $A^{\prime}$ symmetry, instead of the spin-free $a^{3} A^{\prime \prime}$ state. Anisotropy measurements thus fully agree with the SO model (Table 2, Figs. 6 and 7) that assigns the emission to the $\mathrm{CA}^{\prime}$ and (with a lower intensity) $\mathrm{bA}^{\prime}$ states. Contribution of the fourth-state $\mathrm{bA}^{\prime \prime}$ emission could be responsible for the experimental values being slightly lower than 0.4 .

Intersystem crossing. Efficient population of the lowest triplet state in heavy transition metal complexes is another consequence of large SOC. Quantum yields of ${ }^{3}$ MLCT population approach unity because ISC is fast enough to compete with other deactivation processes of the excited singlet. In the spin-free model, a small SOC perturbation is assumed to couple the optically populated ${ }^{1}$ MLCT state with that ${ }^{3}$ MLCT state, which differs in the depopulated d orbital (Section 4.1, rule 4). ISC between such states is allowed because the spin-flip is compensated by dorbital rotation. In the SO-model, ISC is treated as a conversion between SO states. Only few quantitative experimental studies have been published so far, which have revealed fs rates for pseudooctahedral $\mathrm{d}^{6}$ complexes [10,14,15,90,91,120-122] and ps rates for flattened-tetrahedral $\mathrm{d}^{10}$ ones $[93,94]$ and the dimeric $\left[\mathrm{Pt}_{2}\left(\mathrm{P}_{2} \mathrm{O}_{5} \mathrm{H}_{2}\right)_{4}\right]^{4-}[97,99]$. Understanding and controlling ISC kinetics and mechanism remain a challenging problem for inorganic photophysics. A theoretical treatment would require calculating potential energy surfaces of SO states and examining their vibronic coupling.

Ultrafast excited-state dynamics. Pronounced SOC effects could be expected to show up in ultrafast spectroscopic experiments even at ambient temperatures. Closely-spaced low-lying SO states are initially populated from higher optically excited states in a non-equilibrated distribution, each of them vibrationally excited. The high density of SO states will lead to multiple crossings and conical intersections along the decay pathways, affecting or determining excited-state dynamics. One could even imagine creating coherent superpositions of close-lying SO states in heavy-metal complexes upon excitation with fs laser pulses, or a breakdown of Born-Oppenheimer approximation during excited-state evolution.

Generally, electronic relaxation of higher SO states and equilibration of the lower ones, together with vibrational relaxation, will contribute to the kinetics measured by time-resolved spectroscopic techniques on a fs-ps timescale. Because of different sensitivities to individual kinds of relaxation processes and states, we may expect observing different spectroscopic signatures and kinetics in different spectroscopic regions, as well as in absorption and emission. Indeed, complicated ultrafast relaxation dynamics have been observed $[10,14,15,24]$ for $\mathrm{Re}^{\mathrm{I}}$ tricarbonyl diimine complexes, including $\left[\operatorname{Re}(\mathrm{imH})(\mathrm{CO})_{3}(\text { phen })\right]^{+}$, Section 3.3.

\section{Concluding remarks}

Excited-state model based on SO states of a mixed-spin character can be developed for closed-shell heavy metal complexes, using relativistic quantum chemical calculations that explicitly include SOC. The model consists of a single manifold of SO states that replaces the customary sets of singlets and triplets of conventional spin-free models. SO states are characterized by their energies, double-group symmetries, contributing spin-free singlet and triplet states, and the oscillator strengths of corresponding transitions from the ground state. In the case of sufficiently strong SOC, there would be no physical distinction between fluorescence and phosphorescence from SO states, as well as between intersystem crossing and internal conversion. Spin-orbit and vibronic coupling would have to be treated at the same level of theory. The SO-model has important consequences for our understanding of spectroscopic properties and photochemical behavior of heavy metal complexes:

1. SOC tends to broaden and weaken spectroscopic bands relative to those obtained by spin-free calculations. Absorption bands arise from a large number of weaker transitions to SO states, instead of a few intense spin-allowed transitions. SO states of a predominantly triplet origin can significantly contribute to the spectra, if containing small admixtures of strongly allowed singlets. Spectra calculated by the SO- and spin-free techniques are quantitatively similar but, to a considerable extent, of a qualitatively different origin.

2. Emission bands of heavy metal complexes could encompass transitions from several SO states. The lowest SO state usually has the highest triplet parentage and is the least emissive.

3. The large density of SO states allows for multiple electronic and vibrational excitations. Convoluted electronic and vibrational energy redistribution and relaxation are expected to contribute to ultrafast excited-state dynamics. Electronic relaxation will proceed through cascades of state crossings and conical intersections between closely spaced SO states.

4. High-lying SO states are often strongly emissive. Prompt, fast decaying, emission from higher states is expected in heavy metal complexes and ultrafast time-resolved emission studies will provide important photophysical and dynamical information. So far, prompt emission ("fluorescence") from metal complexes has been studied only rarely, see for example Refs. [10,90,93,94,97,99,120-123].

5. Energy of high-lying SO states could be harnessed by ultrafast electron- or energy transfer, provided that electronic relaxation is not competitive. However, the large density of states will generally facilitate deactivation processes and shorten the upper excited-state lifetimes.

6. The high density of SO states could give rise to unexpected photophysical or photochemical phenomena such as coherent population of SO states upon fs excitation, breakdown of Born-Oppenheimer approximation during SO state evolution, stimulated emission from high-lying states, etc. In any case, we should expect seeing manifestations of SO state dynamics in ultrafast experiments. Time-resolved spectroscopy could possibly inform us on the energy separation of the lowest SO states (ZFS) and their equilibration rate in fluid solutions at ambient temperature. The SO-model also predicts excitation-energy dependent photophysics and photochemistry, if a high-lying strongly coupled SO state is selectively excited.

7. The SO model (i.e. relativistic excited-state calculation) of $\left[\operatorname{Re}(\mathrm{imH})(\mathrm{CO})_{3}(\text { phen })\right]^{+}$confirms that the three lowest SO states are largely derived from the lowest spin-free triplet and can be treated as its sublevels. The ZFS concept is thus valid within the SO-model and the experimentally determined ZFS corresponds to the energy separation of the three lowest SO states, as has been indicated by experimental studies of many heavy-metal complexes [1-3]. The qualitative perturbational So approach (Eqs. (4)-(6)) provides a useful guidance on the character and behavior of the lowest-triplet sublevels. However, higher-lying SO states are too mixed to be regarded as sublevels of any specific spin-free triplet.

The relativistic model of SO states provides a conceptual framework to discuss spectroscopic and photophysical phenomena in a unified way. It also presents new theoretical and experimental challenges. Understanding the dynamics and evolution of the dense 
manifolds of SO states will allow us to pinpoint specific deactivation pathways and achieve a better control of photonic behavior of metal complexes.

\section{Acknowledgments}

Support provided by the European COST D35 collaboration program and the Czech Ministry of Education grants LD11086 and ME10124 is gratefully acknowledged. This paper is dedicated to Harry B. Gray, a great scientist and friend.

\section{Appendix A. Computational details}

The electronic structure of $\left[\operatorname{Re}(\mathrm{imH})(\mathrm{CO})_{3}(\text { phen })\right]^{+}$was calculated by DFT and SA-CASSCF/MS-CASPT2 methods using the Gaussian 09 A.02 [124], ADF 2010.01 [125] and MOLCAS 7.4 [70] program packages. The $C_{S}$ constrained symmetry was maintained in all calculations. The DFT methodology included in Gaussian 09 was used for geometry optimization of the ground state and several lowest lying excited states. The geometry of the lowest triplet state was calculated by the UKS approach; the geometry of the $\mathrm{b}^{1} \mathrm{~A}^{\prime}$ state was obtained by TDDFT. DFT calculations employed Perdew, Burke, Ernzerhof (PBE0) hybrid functional [126,127]. The solvent was described by the polarizable conductor calculation model (PCM) [77]. For $\mathrm{H}, \mathrm{C}, \mathrm{N}, \mathrm{O}$, and $\mathrm{Cl}$ atoms, polarized triple$\zeta$ basis sets $6-311 \mathrm{~g}(\mathrm{~d})[128,129]$, together with quasirelativistic effective core pseudopotentials and corresponding optimized set of basis functions for Re $[130,131]$. The bond lengths variation due to the excitation to $\mathrm{b}^{1} \mathrm{~A}^{\prime}$ and $\mathrm{a}^{3} \mathrm{~A}^{\prime \prime}$ states is listed in Table A1.

\section{Table A1}

The DFT (G09/PBE0/PCM-DMF) calculated changes of selected bond lengths of $\left[\operatorname{Re}(\mathrm{imH})(\mathrm{CO})_{3}(\text { phen })\right]^{+}$upon excitation to spin-free $\mathrm{b}^{1} \mathrm{~A}^{\prime}$ and $\mathrm{a}^{3} \mathrm{~A}^{\prime \prime}$ states.

\begin{tabular}{lcc}
\hline Bond & $\Delta r\left(\mathrm{~b}^{1} \mathrm{~A}^{\prime}-\mathrm{GS}\right)$ & $\Delta r\left(\mathrm{a}^{3} \mathrm{~A}^{\prime \prime}-\mathrm{GS}\right)$ \\
\hline $\mathrm{Ru}-\mathrm{N}$ & -0.062 & -0.077 \\
$\mathrm{Ru}-\mathrm{N}(\mathrm{im})$ & -0.028 & -0.079 \\
$\mathrm{Ru}-\mathrm{C}(\mathrm{ax})$ & 0.032 & 0.083 \\
$\mathrm{Ru}-\mathrm{C}(\mathrm{eq})$ & 0.053 & 0.049 \\
$\mathrm{~N}-\mathrm{C} 2$ & 0.026 & 0.032 \\
$\mathrm{C} 2-\mathrm{C2}$ & -0.028 & -0.037 \\
\hline
\end{tabular}

The SO-RASSI calculations were performed by the MOLCAS 7.4 software. 18 electrons have been correlated in 14 active orbitals ( 7 of the $A^{\prime}$ symmetry, 7 of the $A^{\prime \prime}$ symmetry). Six ${ }^{1} A^{\prime}$, four ${ }^{1} A^{\prime \prime}$, five ${ }^{3} A^{\prime}$ and five ${ }^{3} A^{\prime \prime}$ spin-free states have been included into the calculation, resulting in 40 spin-orbit states. For all the atoms, atomic natural orbitals relativistic consistent correlated ANO-RCC basis sets have been used with the following double-zeta contraction scheme: (8s, $4 \mathrm{p}, 3 \mathrm{~d}, 1 \mathrm{f})$ contracted to [2s, 1p] for $\mathrm{H},(14 \mathrm{~s}, 9 \mathrm{p}, 4 \mathrm{~d}, 3 \mathrm{f}, 2 \mathrm{~g})$ contracted to $[3 \mathrm{~s}, 2 \mathrm{p}, 1 \mathrm{~d}]$ for $\mathrm{C}, \mathrm{N}, \mathrm{O}$ and triple-zeta contraction scheme (24s, $21 \mathrm{p}, 15 \mathrm{~d}, 11 \mathrm{f}, 4 \mathrm{~g}, 2 \mathrm{~h}$ ) contracted to [7s, 6p, 4d, 2f, 1g] for the rhenium atom.

The ADF program first performed scalar relativistic TDDFT calculations in order to determine the lowest single-group symmetry excited states and then the spin-orbit coupling operator was applied to obtain excitation energies with spin-orbit coupling effects included [73]. Slater type orbital (STO) basis sets of triple- $\zeta$ quality with two polarization functions for Ru and double- $\zeta$ quality with one polarization function for remaining atoms were employed (core electrons were included). PBE0 hybrid functional together with the scalar relativistic (SR) zero order regular approximation (ZORA) [132] was used within this study. COSMO [76] model was used for the modeling of the solvent effect.

\section{References}

[1] H. Yersin, W.J. Finkenzeller, in: H. Yersin (Ed.), Highly Efficient OLEDs with Phosphorescent Materials, Wiley-VCH, Weinheim, 2008, p. 1.

[2] A.F. Rausch, H.H.H. Homeier, H. Yersin, Top. Organomet. Chem. 29 (2010) 193

[3] H. Yersin, A.F. Rausch, R. Czerwieniec, T. Hofbeck, T. Fischer, Coord. Chem. Rev 255 (2011) (in press).

[4] G.A. Crosby, K.W. Hipps, W.H.J. Elfring, J. Am. Chem. Soc. 96 (1974) 629.

[5] F. Neese, T. Petrenko, D. Ganyushin, G. Olbrich, Coord. Chem. Rev. 251 (2007) 288.

[6] C. Daniel, Coord. Chem. Rev. 238-239 (2003) 143.

[7] C. Daniel, Coord. Chem. Rev. 230 (2002) 65.

[8] M. Turki, C. Daniel, S. Záliš, A. Vlček Jr., J. van Slageren, D.J. Stufkens, J. Am. Chem. Soc. 123 (2001) 11431.

[9] A. Vlček Jr., S. Záliš, Coord. Chem. Rev. 251 (2007) 258.

[10] A. Cannizzo, A.M. Blanco-Rodríguez, A. Nahhas, J. Šebera, S. Záliš, A. Vlček Jr. M. Chergui, J. Am. Chem. Soc. 130 (2008) 8967.

[11] A.M. Blanco-Rodríguez, A.J. Di Bilio, C. Shih, A.K. Museth, I.P. Clark, M. Towrie A. Cannizzo, J. Sudhamsu, B.R. Crane, J. Sýkora, J.R. Winkler, H.B. Gray, S. Záliš, A. Vlček Jr., Chem. Eur. J 17 (2011) (in press).

[12] A.M. Blanco-Rodríguez, A. Gabrielsson, M. Motevalli, P. Matousek, M. Towrie J. Šebera, S. Záliš, A. Vlček Jr., J. Phys. Chem. A 109 (2005) 5016.

[13] A. Gabrielsson, M. Busby, P. Matousek, M. Towrie, E. Hevia, L. Cuesta, J. Perez, S. Záliš, A. Vlček Jr., Inorg. Chem. 45 (2006) 9789.

[14] A. El Nahhas, C. Consani, A.M. Blanco-Rodríguez, K.M. Lancaster, O. Braem, A. Cannizzo, M. Towrie, I.P. Clark, S. Záliš, M. Chergui, A. Vlček Jr., Inorg. Chem. 50 (2011) (in press).

[15] A. El Nahhas, A. Cannizzo, F. van Mourik, A.M. Blanco-Rodríguez, S. Záliš, A. Vlček Jr., M. Chergui, J. Phys. Chem. A 114 (2010) 6361.

[16] G.S.-M. Tong, C.-M. Che, Chem. Eur. J. 15 (2009) 7225.

[17] S. Obara, M. Itabashi, F. Okuda, S. Tamaki, Y. Tanabe, Y. Ishii, K. Nozaki, M.-a. Haga, Inorg. Chem. 45 (2006) 8907.

[18] V. Vallet, A. Strich, C. Daniel, Chem. Phys. 311 (2005) 13.

[19] M. Kayanuma, C. Daniel, H. Köppel, E. Gindensperger, Coord. Chem. Rev. 255 (2011) (in press)

[20] C.A. Ndoye, C. Daniel, Chin. J. Chem. Phys. 22 (2009) 171

[21] D.J. Stufkens, A. Vlček Jr., Coord. Chem. Rev. 177 (1998) 127.

[22] A. Vlček Jr., Top. Organomet. Chem. 29 (2010) 73.

[23] A. Kumar, S.-S. Sun, A.J. Lees, Top. Organomet. Chem. 29 (2010) 1.

[24] D.J. Liard, M. Busby, P. Matousek, M. Towrie, A. Vlček Jr., J. Phys. Chem. A 108 (2004) 2363.

[25] C. Shih, A.K. Museth, M. Abrahamsson, A.M. Blanco-Rodriguez, A.J. Di Bilio, J Sudhamsu, B.R. Crane, K.L. Ronayne, M. Towrie, A. Vlček Jr., J.H. Richards, J.R Winkler, H.B. Gray, Science 320 (2008) 1760.

[26] A.M. Blanco-Rodríguez, M. Towrie, S. Záliš, J. Sýkora, A. Vlček, Jr. Inorg. Chem. (submitted for publication).

[27] P. Chen, T.D. Westmoreland, E. Danielson, K.S. Schanze, D. Anthon, P.E. Neveux Jr., T.J. Meyer, Inorg. Chem. 26 (1987) 1116.

[28] J.D. Lewis, L. Bussotti, P. Foggi, R.N. Perutz, J.N. Moore, J. Phys. Phem. A 106 (2002) 12202.

[29] S.Y. Reece, D.G. Nocera, J. Am. Chem. Soc. 127 (2005) 9448.

[30] J. Hawecker, J.-M. Lehn, R. Ziessel, J. Chem. Soc., Chem. Commun. (1983) 536

[31] H. Takeda, K. Koike, H. Inoue, O. Ishitani, J. Am. Chem. Soc. 130 (2008) 2023.

[32] H. Takeda, O. Ishitani, Coord. Chem. Rev. 254 (2010) 346.

[33] K.K.-W. Lo, Top. Organomet. Chem. 29 (2010) 115

[34] K.K.-W. Lo, K.-S. Sze, K.H.-K. Tsang, N. Zhu, Organometallics 26 (2007) 3440.

[35] K.K.-W. Lo, K.H.-K. Tsang, K.-S. Sze, C.-K. Chung, T.K.-M. Lee, K.Y. Zhang, W.-K. Hui, C.-K. Li, J.S.-Y. Lau, D.C.-M. Ng, N. Zhu, Coord. Chem. Rev. 251 (2007) 2292.

[36] K.K.-W. Lo, M.-W. Louie, K.Y. Zhang, Coord. Chem. Rev. 254 (2010) 2603.

[37] A.F.A. Peacock, H.D. Batey, C. Raendler, A.C. Whitwood, R.N. Perutz, A.-K. Duhme-Klair, Angew. Chem. Int. Ed. 44 (2005) 1712

[38] J.D. Lewis, J.N. Moore, Dalton Trans. (2004) 1376

[39] C.-C. Ko, W.-M. Kwok, V.W.-W. Yam, D.L. Phillips, Chem. Eur. J. 12 (2006) 5840

[40] A.O.T. Patrocinio, N.Y.M. Iha, Inorg. Chem. 47 (2008) 10851.

[41] A.S. Sarto Polo, M.K. Itokazu, K.M. Frin, A.O. de Toledo Patrocinio, N.Y.M. Iha, Coord. Chem. Rev. 250 (2006) 1669.

[42] A. Beyeler, P. Belser, L. De Cola, Angew. Chem. Int. Ed. Engl. 36 (1997) 2779.

[43] D.M. Cleland, G. Irwin, P. Wagner, D.L. Officer, K.C. Gordon, Chem. Eur. J. 15 (2009) 3682.

[44] W.B. Connick, A.J. Di Bilio, M.G. Hill, J.R. Winkler, H.B. Gray, Inorg. Chim. Acta 240 (1995) 169.

[45] B.R. Crane, A.J. Di Bilio, J.R. Winkler, H.B. Gray, J. Am. Chem. Soc. 123 (2001) 11623.

[46] A.J. Di Bilio, B.R. Crane, W.A. Wehbi, C.N. Kiser, M.M. Abu-Omar, R.M. Carlos J.H. Richards, J.R. Winkler, H.B. Gray, J. Am. Chem. Soc. 123 (2001) 3181.

[47] J.E. Miller, C. Gradinaru, B.R. Crane, A.J. Di Bilio, W.A. Wehbi, S. Un, J.R. Winkler, H.B. Gray, J. Am. Chem. Soc. 125 (2003) 14220.

[48] J.E. Miller, A.J. Di Bilio, W.A. Wehbi, M.T. Green, A.K. Museth, J.R. Richards, J.R. Winkler, H.B. Gray, Biochim. Biophys. Acta 1655 (2004) 59.

[49] C. Grădinaru, B.R. Crane, J. Phys. Chem. B 110 (2006) 20073.

[50] A.M. Blanco-Rodríguez, M. Busby, C. Grădinaru, B.R. Crane, A.J. Di Bilio, P. Matousek, M. Towrie, B.S. Leigh, J.H. Richards, A. Vlček Jr., H.B. Gray, J. Am. Chem. Soc. 128 (2006) 4365.

[51] A.M. Blanco-Rodríguez, M. Busby, K.L. Ronayne, M. Towrie, J. Sýkora, M. Hof S. Záliš, C. Grădinaru, A.J. Di Bilio, B.R. Crane, H.B. Gray, J. Vlček, J. Am. Chem. Soc. 131 (2009) 11788. 
[52] W. Belliston-Bittner, A.R. Dunn, Y.H. Le Nguyen, D.J. Stuehr, J.R. Winkler, H.B. Gray, J. Am. Chem. Soc. 127 (2005) 15907.

[53] A.R. Dunn, W. Belliston-Bittner, J.R. Winkler, E.D. Getzoff, D.J. Stuehr, H.B. Gray, J. Am. Chem. Soc. 127 (2005) 5169.

[54] Y.H. Le Nguyen, J.R. Winkler, H.B. Gray, J. Phys. Chem. B 111 (2007) 6628.

[55] M.W. George, F.P.A. Johnson, J.R. Westwell, P.M. Hodges, J.J. Turner, J. Chem. Soc., Dalton Trans. (1993) 2977.

[56] D.R. Gamelin, M.W. George, P. Glyn, F.-W. Grevels, F.P.A. Johnson, W. Klotzbücher, S.L. Morrison, G. Russell, K. Schaffner, J.J. Turner, Inorg. Chem. 33 (1994) 3246.

[57] D.M. Dattelbaum, K.M. Omberg, J.R. Schoonover, R.L. Martin, T.J. Meyer, Inorg. Chem. 41 (2002) 6071.

[58] D.M. Dattelbaum, K.M. Omberg, P.J. Hay, N.L. Gebhart, R.L. Martin, J.R. Schoonover, T.J. Meyer, J. Phys. Chem. A 108 (2004) 3527.

[59] D.M. Dattelbaum, R.L. Martin, J.R. Schoonover, T.J. Meyer, J. Phys. Chem. A 108 (2004) 3518.

[60] B.D. Rossenaar, D.J. Stufkens, A. Vlček Jr., Inorg. Chem. 35 (1996) 2902.

[61] A. Vlček Jr., S. Záliš, J. Phys. Chem. A 109 (2005) 2991.

[62] S. Záliš, N. Ben Amor, C. Daniel, Inorg. Chem. 43 (2004) 7978.

[63] A.M. Blanco-Rodríguez, K.L. Ronayne, S. Záliš, J. Sýkora, M. Hof, A. Vlček Jr., J. Phys. Chem. A 112 (2008) 3506.

[64] A.M. Blanco-Rodríguez, M. Towrie, J.-P. Collin, S. Záliš, A. Vlček Jr., Dalton Trans. (2009) 3941.

[65] A.M. Blanco-Rodríguez, M. Towrie, I.P. Clark, S. Záliš, A. Vlček, Jr. (in preparation).

[66] I.P. Clark, M.W. George, F.P.A. Johnson, J.J. Turner, Chem. Commun. (1996) 1587.

[67] P. Atkins, R. Friedman, Molecular Quantum Mechanics, Oxford University Press, Oxford, 2011.

[68] N. Kaltsoyannis, J. Chem. Soc., Dalton Trans. (1997) 1.

[69] P. Pyykkö, Chem. Rev. 88 (1988) 563.

[70] G. Karlström, R. Lindh, P.-Å. Malmqvist, B.O. Roos, U. Ryde, V. Veryazov, P.-O. Widmark, M. Cossi, B. Schimmelpfennig, P. Neogrady, L. Seijo, Comput. Mater. Sci. 28 (2003) 222.

[71] B.O. Roos, P.-Å. Malmqvist, Phys. Chem. Chem. Phys. 6 (2004) 2919.

[72] C.M. Marian, U. Wahlgren, Chem. Phys. Lett. 251 (1996) 357.

[73] F. Wang, T. Ziegler, J. Chem. Phys. 123 (2005) 154102.

[74] G. te Velde, F.M. Bickelhaupt, S.J.A. van Gisbergen, C. Fonseca Guerra, E.J. Baerends, J.G. Snijders, T. Ziegler, J. Comput. Chem. 22 (2001) 931.

[75] A. Klamt, V. Jones, J. Chem. Phys. 105 (1996) 9972.

[76] A. Klamt, G. Schüürmann, J. Chem. Soc., Perkin Trans. 2 (1993) 799.

[77] J. Tomasi, B. Mennucci, R. Cammi, Chem. Rev. 105 (2005) 2999.

[78] F. Wang, T. Ziegler, E. van Lenthe, S. van Gisbergen, E.J. Baerends, J. Chem. Phys. 122 (2005) 204103.

[79] E. van Lenthe, J.G. Snijders, E.J. Baerends, J. Chem. Phys. 105 (1994) 6505.

[80] M. Busby, A. Gabrielsson, P. Matousek, M. Towrie, A.J. Di Bilio, H.B. Gray, A. Vlček Jr., Inorg. Chem. 43 (2004) 4994.

[81] L.A. Worl, R. Duesing, P. Chen, L. Della Ciana, T.J. Meyer, J. Chem. Soc., Dalton Trans. (1991) 849.

[82] L. Wallace, D.C. Jackman, D.P. Rillema, J.W. Merkert, Inorg. Chem. 34 (1995) 5210.

[83] D.R. Striplin, G.A. Crosby, Chem. Phys. Lett. 221 (1994) 426.

[84] D.R. Striplin, G.A. Crosby, Coord. Chem. Rev. 211 (2001) 163.

[85] J. Van Houten, R.J. Watts, Inorg. Chem. 17 (1978) 3381.

[86] J. Van Houten, R.J. Watts, J. Am. Chem. Soc. 98 (1976) 4853

[87] F. Alary, J.-L. Heully, L. Bijeire, P. Vicendo, Inorg. Chem. 46 (2007) 3154.

[88] S. Záliš, M. Busby, T. Kotrba, P. Matousek, M. Towrie, A. Vlček Jr., Inorg. Chem. 43 (2004) 1723.

[89] S. Záliš, I.R. Farrell, A. Vlček Jr., J. Am. Chem. Soc. 125 (2003) 4580.

[90] W. Gawelda, A. Cannizzo, V.-T. Pham, F. van Mourik, C. Bressler, M. Chergui, J. Am. Chem. Soc. 129 (2007) 8199.

[91] C. Consani, M. Mirabelle Prémont-Schwarz, A. El Nahhas, C. Bressler, F. van Mourik, A. Cannizzo, M. Chergui, Angew. Chem. Int. Ed. 48 (2009) 7184.

[92] J.N. Schrauben, K.L. Dillman, W.F. Beck, J.K. McCusker, Chem. Sci. 1 (2010) 405.

[93] Z.A. Siddique, Y. Yamamoto, T. Ohno, K. Nozaki, Inorg. Chem. 42 (2003) 6366.
[94] Z.A. Siddique, T. Ohno, K. Nozaki, Inorg. Chem. 43 (2004) 663.

[95] C.J. Ballhausen, Introduction to Ligand Field Theory, McGraw-Hill, Inc., New York, 1962.

[96] I.V. Khudyakov, Y.A. Serebrennikov, N.J. Turro, Chem. Rev. 93 (1993) 537.

[97] S.J. Milder, B.S. Brunschwig, J. Phys. Chem. 96 (1992) 2189.

[98] Y. Shimizu, Y. Tanaka, T. Azumi, J. Phys. Chem. 88 (1984) 2423.

[99] R.M. van der Veen, A. Cannizzo, F. van Mourik, A. Vlček Jr., M. Chergui, J. Am. Chem. Soc. 133 (2011) 305.

[100] K. Nozaki, J. Chin. Chem. Soc. 53 (2006) 101.

[101] E. Jansson, B. Minaev, S. Schrader, H. Ågren, Chem. Phys. 333 (2007) 157.

[102] E.M. Kober, T.J. Meyer, Inorg. Chem. 21 (1982) 3967.

[103] E.M. Kober, T.J. Meyer, Inorg. Chem. 23 (1984) 3877.

[104] J. Ferguson, F. Herren, Chem. Phys. 76 (1983) 45.

[105] Y. Komada, S. Yamauchi, N. Hirota, J. Phys. Chem. 90 (1986) 6425.

[106] H. Miki, M. Shimada, T. Azumi, J.A. Brozik, G.A. Crosby, J. Phys. Chem. 97 (1993) 11175.

[107] C. Daul, E.J. Baerends, P. Vernooijs, Inorg. Chem. 33 (1994) 3538.

[108] C. Daniel, M.-C. Heitz, J. Manz, C. Ribbing, J. Chem. Phys. 102 (1995) 905.

[109] C. Daniel, D. Guillaumont, C. Ribbing, B. Minaev, J. Phys. Chem. A 103 (1999) 5766.

[110] E. Gindensperger, H. Köppel, C. Daniel, Chem. Commun. 46 (2010) 8225.

[111] T. Matsushita, T. Asada, S. Koseki, J. Phys. Chem. A 110 (2006) 13295.

[112] B. Minaev, V. Minaeva, H. Ågren, J. Phys. Chem. A 113 (2009) 726.

[113] W.J. Finkenzeller, T. Hofbeck, M.E. Thompson, H. Yersin, Inorg. Chem. 46 (2007) 5076

[114] P. Chen, T.J. Meyer, Chem. Rev. 98 (1998) 1439.

[115] A.F. Rausch, U.V. Monkowius, M.Zabel, H. Yersin, Inorg. Chem. 49 (2010) 7818.

[116] T. Hofbeck, H. Yersin, Inorg. Chem. 49 (2010) 9290.

[117] R. Czerwieniec, T. Hofbeck, O. Crespo, A. Laguna, M. Concepcion Gimeno, H. Yersin, Inorg. Chem. 49 (2010) 3764.

[118] L. Sokolová, H. Williamson, J. Sýkora, M. Hof, H.B. Gray, B. Brutschy, A. Vlček, Jr. (in preparation).

[119] X.-Q. Guo, F.N. Castellano, L. Li, J.R. Lakowicz, Anal. Chem. 70 (1998) 632.

[120] A. Cannizzo, F. van Mourik, W. Gawelda, G. Zgrablic, C. Bressler, M. Chergui, Angew. Chem. Int. Ed. 45 (2006) 3174

[121] A.C. Bhasikuttan, T. Okada, J. Phys. Chem. B 108 (2004) 12629.

[122] G. Hedley, A. Ruseckas, I.D.W. Samuel, J. Phys. Chem. A 113 (2009) 2.

[123] A.C. Bhasikuttan, M. Suzuki, S. Nakashima, T. Okada, J. Am. Chem. Soc. 124 (2002) 8398.

[124] M.J. Frisch, G.W. Trucks, H.B. Schlegel, G.E. Scuseria, M.A. Robb, J.R. Cheeseman, G. Scalmani, V. Barone, B. Mennucci, G.A. Petersson, H. Nakatsuji, M. Caricato, X. Li, H.P. Hratchian, A.F. Izmaylov, J. Bloino, G. Zheng, J.L. Sonnenberg, M. Hada, M. Ehara, K. Toyota, R. Fukuda, J. Hasegawa, M. Ishida, T. Nakajima, Y. Honda, O. Kitao, H. Nakai, T. Vreven, J.A. Montgomery Jr., J.E. Peralta, F. Ogliaro, M. Bearpark, J.J. Heyd, E. Brothers, K.N. Kudin, V.N. Staroverov, R. Kobayashi, J. Normand, K. Raghavachari, A. Rendell, J.C. Burant, S.S. Iyengar, J. Tomasi, M. Cossi, N. Rega, J.M. Millam, M. Klene, J.E. Knox, J.B. Cross, V. Bakken, C. Adamo, J. Jaramillo, R. Gomperts, R.E. Stratmann, O. Yazyev, A.J. Austin, R. Cammi, C. Pomelli, J.W. Ochterski, R.L. Martin, K. Morokuma, V.G. Zakrzewski, G.A. Voth, P. Salvador, J.J. Dannenberg, S. Dapprich, A.D. Daniels, O. Farkas, J.B. Foresman, J.V. Ortiz, J. Cioslowski, D.J. Fox, Gaussian 09, Revision A.02, Gaussian, Inc., Wallingford, CT, 2009.

[125] C. Fonseca Guerra, J.G. Snijders, G. te Velde, E.J. Baerends, Theor. Chim. Acta 99 (1998) 391.

[126] J.P. Perdew, K. Burke, M. Ernzerhof, Phys. Rev. Lett. 77 (1996) 3865.

[127] C. Adamo, V. Barone, J. Chem. Phys. 110 (1999) 6158.

[128] K. Raghavachari, J.S. Binkley, R. Seeger, J.A. Pople, J. Chem. Phys. 72 (1980) 650.

[129] L.A. Curtiss, M.P. McGrath, J.-P. Blaudeau, N.E. Davis, R.C. Binning Jr., L. Radom, J. Chem. Phys. 103 (1995) 6104.

[130] D. Andrae, U. Häussermann, M. Dolg, H. Stoll, H. Preuss, Theor. Chim. Acta 77 (1990) 123.

[131] J.M.L. Martin, A. Sundermann, J. Chem. Phys. 114 (2001) 3408.

[132] E. van Lenthe, A. Ehlers, E.J. Baerends, J. Chem. Phys. 110 (1999) 8943. 